\title{
Les actes délégués dans le système des sources du droit de l'Union Européenne
}

\section{Delegated Acts in the System of Sources of European Union \\ Law}

\section{Gregorio Garzón Clariana}

(C) ERA 2011

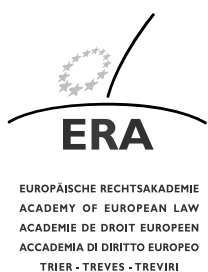

Résumé Jurisconsulte honoraire du Parlement européen, l'auteur propose ici une contribution importante à l'étude des sources du droit de l'Union européenne, à travers une analyse approfondie et renouvelée de la nouvelle catégorie d'acte consacrée par le Traité de Lisbonne, celles des actes délégués. L'accent est mis sur les aspects relatifs à l'application et à l'interprétation des nouvelles dispositions de l'article 290 TFUE.

Mots clés Article 290 TFUE · Comitologie · Décision du Conseil 2006/512/CE . Pouvoir législatif · Institutions de l'UE · Convention européenne · Traité de Lisbonne

Abstract In this article the author - Honorary Jurisconsulte of the European Parliament - provides a thorough and revealing analysis of the sources of law of the European Union, focusing on the new category of legal act created by the Treaty of Lisbon, namely delegated acts, and in particular on aspects relating to the application and interpretation of the new provisions in Article 290 TFEU.

Keywords Article 290 TFEU · Comitology • Council Decision 2006/512/EC · European Convention · Institutions of the EU · Legislative power · Lisbon Treaty

Professeur ordinaire (Catedrático) de Droit International Public à l'Université Autonome de Barcelone; Chaire Jean Monnet ad personam d'Études Juridiques de l'Union européenne ; Membre d'honneur du Comité consultatif de l'Académie de droit européen (ERA). Une version antérieure de ce travail a été publiée comme Étude dans la Revista de Derecho Comunitario Europeo 37 (2010), pp. 721-760.

Prof. G. Garzón Clariana $(\varangle)$

Universitat Autònoma de Barcelona, Bellaterra, Barcelona, Spain

e-mail: Gregorio.Garzon@uab.cat 


\section{Introduction}

La consécration des actes délégués, bien connus dans le droit comparé, comme nouvelle catégorie d'actes juridiques dans le système des sources du Droit de l'Union Européenne avait été accueillie comme une des innovations importantes du Traité constitutionnel, ${ }^{1}$ faisant bientôt l'objet de commentaires du point de vue de ce qui semblait alors une «constitutionnalisation» imminente de la construction européenne par des auteurs appartenant à différentes disciplines juridiques.

La longue période d'incertitude sur l'avenir du Traité constitutionnel, prolongée en raison des difficultés qu'a posé l'entrée en vigueur du Traité de Lisbonne qui devait venir le remplacer, explique sans nul doute que ces premières réflexions individuelles de la doctrine juridique n'aient pas été suivies de recherches de plus grande portée ou d'une véritable discussion doctrinale, malgré de nouveaux développements de la jurisprudence et des pratiques d'un indéniable intérêt.

Le but de ce travail est de contribuer à l'étude du système des sources du Droit de l'Union Européenne, à travers une reconsidération actualisée et une analyse plus développée des actes délégués à la lumière des travaux préparatoires de la Convention européenne et des deux Conférences intergouvernementales l'ayant suivie, des développements intervenus après la signature du Traité constitutionnel, du nouveau contexte du Traité de Lisbonne (y compris ses Protocoles) et de la première année d'expérience dans l'application des nouvelles dispositions introduites dans les Traités.

L'accent sera mis sur les aspects relatifs à l'application et à l'interprétation des nouvelles dispositions, plutôt que sur des préférences de iure condendo qui, bien que tout à fait légitimes, semblent maintenant moins opportunes. Cependant, cela n'est pas incompatible avec une perspective éprise de légitimité démocratique dans l'Union Européenne, qui pourrait difficilement être évitée en présence d'une catégorie d'actes juridiques qui trouve justement sa raison d'être dans le besoin de concilier les exigences d'un débat parlementaire digne de ce nom, avec l'efficacité que la citoyenneté doit pouvoir exiger de tous les pouvoirs publics. ${ }^{2}$

\section{Les règlements délégués dans le Traité constitutionnel et la délégation dans le droit communautaire ultérieur}

Les actes délégués n'étaient pas prévus dans l'article 40 du projet Spinelli de 1984, plutôt inspiré du principe d'un « pouvoir réglementaire » propre à la Commission européenne, comme le confirme l'article 28 du même projet lorsque, parmi les fonctions de cette Institution, il fait mention de celle de prendre des « règlements d'application des lois » $;^{3}$ ils font leur apparition plus tard, dans les contributions suivantes apportées par le Parlement européen ou par sa commission chargée des relations institu-

\footnotetext{
${ }^{1}$ Cf. e.g. Piris [48], pp. 73-76.

${ }^{2}$ La présente étude fait partie de la recherche prévue dans le projet « Démocratie et Droit dans les Institutions européennes » de la Chaire Jean Monnet ad personam de l'auteur.

${ }^{3}$ Parlement européen : Projet de Traité instituant l'Union européenne, Luxembourg 1984.
} 
tionnelles, avant les élections du 15 juin 1994 (les rapports Colombo, le projet Oreja et le rapport Hermann ${ }^{4}$ ).

Néanmoins, ce changement d'orientation n'avait pas eu de conséquences directes sur la Convention européenne, conditionnée par des développements plus récents, de sorte qu'il en constitue plus un antécédent que l'origine même des « Règlements délégués ». 5

\subsection{La genèse des « règlements délégués » dans la Convention européenne}

Les travaux de la Convention européenne constituent une source d'informations riche et semblent également être importants pour l'établissement du sens des dispositions du Traité constitutionnel reprises par le Traité de Lisbonne, puisque, d'après la jurisprudence, la Cour de Justice estime applicables aux Traités dudit « Droit primaire » les dispositions de la Convention de Vienne sur le Droit des Traités du 23 mai 1969, relatives à l'interprétation des Traités, parmi lesquelles se trouve celle qui reconnaît aux travaux préparatoires une valeur comme moyen complémentaire d'interprétation. ${ }^{6}$ Dans le contexte de l'Union européenne, dont le fonctionnement « est basé sur la démocratie représentative $»,{ }^{7}$ il serait paradoxal de nier l'importance des travaux d'une Convention composée de représentants de Parlements et de Gouvernements démocratiques, qui est à l'origine de l'immense majorité des nouvelles dispositions des Traités constitutifs de l'Union.

Dans le cas du régime juridique des actes délégués, il semble indispensable de connaître les travaux pertinents de la Convention européenne, compte tenu du fait que les dispositions du Traité de Lisbonne conservent pratiquement la rédaction de la Convention. ${ }^{8}$

\subsubsection{La ratio legis de l'introduction des règlements délégués}

Les profils innovateurs des actes délégués dans les dispositions du Traité constitutionnel ont été critiqués comme étant le fruit d'une décision "pas très méditée », parfait exemple d'une «mauvaise technique législative » qui obéirait à une obsession d'imiter le Droit étatique. ${ }^{9}$ Mais les documents pertinents ne confirment absolument pas cette impression.

\footnotetext{
${ }^{4}$ Sur le sens de cette étape, cf. e.g. Carreras [11], p. 213.

${ }^{5}$ À titre d'exemple, les actes délégués n'étaient pas visés par le projet de Basic Treaty of the European Union soumis par le Centre Robert Schuman de l'Institut Universitaire européen en 2000.

${ }^{6}$ Avis 1/91 du 14 décembre 1991, Rec. p. I-6079, paragraphe 14. Ce paragraphe fait précisément application de la «règle générale d'interprétation » et non pas des moyens complémentaires, mais rien ne laisse supposer que la Cour ait voulu n'adopter que partiellement le système d'interprétation de la Convention de Vienne, écartant le recours auxdits moyens.

${ }^{7}$ Article 10, paragraphe 1, du Traité de l'Union européenne.

${ }^{8}$ Pour une présentation plus étendue des travaux relatifs aux actes juridiques, voir Rosado Pacheco [52], pp. 335-370; pp. 339-351.

${ }^{9} \mathrm{La}$ critique mentionnée dans le texte semble provenir en partie d'une lecture pour le moins discutable de la référence à un épisode (la réunion du Præsidium du 14 février 2003) dans un ouvrage publié par un de ses protagonistes : voir Gil Ibáñzez [20], pp. 63-79; pp. 74, 75 et 79.
} 
Les actes délégués n'étaient pas expressément visés dans le schéma des articles de l'«Avant-projet de Traité constitutionnel » présenté par le Président Valéry Giscard d'Estaing, en octobre $2002 ;{ }^{10}$ en effet, ils émanent d'une initiative du Groupe de travail IX, présidé par le Vice-Président Giuliano Amato et consacré à la « simplification », ainsi que de réflexions concordantes au sein du Parlement européen.

Le Groupe IX avait estimé que son mandat ne se limitait pas à une simplification technique ou formelle, mais qu'il comprenait également l'amélioration de la légitimité démocratique et l'efficacité pratique des instruments juridiques de l'Union, ce qui l'avait amené à tenir compte d'aspects tels que la séparation des pouvoirs, la hiérarchie des normes juridiques ou la notion même de législation ; mais il s'est centré sur la distinction entre les actes législatifs et les actes non législatifs seulement suite à l'audition d' «experts juridiques », au début du mois de novembre 2002, surtout grâce à l'insistance de l'un des experts invités, le Professeur Koen Lenaerts, ${ }^{11}$ alors juge du Tribunal de première instance des Communautés européennes. Peu après, le Secrétariat a proposé une distinction entre trois types d'actes : les actes législatifs, les actes délégués et les actes d'exécution.

Le rapport final du Groupe, en date du 29 novembre 2002, affirmait qu' "il n'y a rien de plus compliqué que la simplification », car cette dernière avait de considérables répercussions «ayant un rapport direct avec le niveau de démocratie » des Institutions de l'Union. ${ }^{12}$ Il précisait également qu'il avait essayé de rendre le système de l'Union plus « lisible», et de «garantir que des actes de même force juridique et politique aient le même fondement quant à leur légitimité démocratique », ce qui avait « directement conduit à une hiérarchie plus nette des normes, comme conséquence d'une meilleure séparation des pouvoirs ». ${ }^{13}$

De même, il était nécessaire de tenter d'éviter l'excès de détail de la législation européenne, critiqué dans les États membres ${ }^{14}$ et dans la Convention européenne elle-même, aux fins de faciliter son adaptation à un environnement changeant, en particulier dans certains domaines économiques. Or, dans la mesure où la possibilité de régler cette question par la voie de la limitation des prérogatives du législateur et par l'extension du recours à la « comitologie» aurait été difficilement conciliable avec les considérations qui préconisaient la généralisation de la procédure de codécision, le Groupe avait dû se tourner vers la recherche d'une nouvelle catégorie d'actes et avait fini par choisir celle des « règlements délégués ». ${ }^{15}$

Les actes délégués font donc leur apparition dans le rapport final du Groupe IX comme une catégorie intermédiaire entre les actes législatifs et les actes d'exécution. À la différence des actes législatifs (qui trouvent leur fondement juridique directement dans le Traité et qui «contiennent les éléments essentiels et les options politiques fondamentales dans un domaine déterminé »), les « règlements délégués »

\footnotetext{
${ }^{10}$ Cf. CONV 369/02 du 28 octobre 2002, articles 28 à 32. La documentation pertinente de la Convention européenne peut encore être consultée sur le site web http ://european-convention.eu.int.

${ }^{11}$ Cf. Liisberg [32], pp. 12-14.

${ }^{12}$ Rapport final du Groupe IX «Simplification », CONV 424/02, p. 1.

${ }^{13}$ Loc. cit. pp. 1-2.

${ }^{14}$ Cf. Kellerman [29]; Durand [18], pp. 41-52.

${ }^{15}$ Cf. e.g. Carrera Hernández [10], p. 1041 ; p. 1053.
} 
sont conçus comme le résultat de l'exercice d'un pouvoir de délégation du législateur, qui «peut aller de la réglementation des éléments techniques ou détaillés qui développent un acte législatif à l'adaptation ultérieure de certains éléments de l'acte législatif lui-même ».

Cette position du Groupe IX coïncidait avec la réflexion au sein du Parlement européen de trouver la façon d'éviter que l'amélioration de la qualité de la législation se fasse au détriment des prérogatives de l'Institution, en limitant la légitimité démocratique du Droit issu de l'Union. Dans ce contexte, il faut mentionner (même si la doctrine semble les ignorer) les délibérations officieuses de l'intergroupe Kangourou avec un Groupe de travail sur la «modernisation de la législation de l'UE », dont les réunions ont eu lieu entre avril et octobre 2002, sous la présidence de Karl von Wogau et avec la participation de députés influents tels que MM. Bourlanges, Hermann et Medina Ortega. ${ }^{16} \mathrm{Au}$ terme de ces travaux, on avait fait remarquer que le projet alors en préparation au sein de la commission chargée des relations constitutionnelles, justement confié à M. Bourlanges, reconnaissait un trop grand pouvoir réglementaire à l'exécutif et semblait trouver son inspiration dans la Constitution de la $\mathrm{V}^{\mathrm{e}}$ République française et, par conséquent, en définitive, dans les idées du Général De Gaulle, limitatrices du rôle de l'Assemblée Nationale. ${ }^{17}$

Toujours est-il que tant le rapport final de la commission chargée des questions constitutionnelles du Parlement européen que la Résolution ultérieure adoptée en session plénière, en décembre 2002, bien que n'abandonnant pas formellement l'idée d'un pouvoir réglementaire propre à la Commission européenne, venaient la compléter par la mention de modalités, de limites et de mesures de contrôle à définir par les deux branches du pouvoir législatif (le Parlement européen et le Conseil). ${ }^{18}$ Cela la différenciait nettement du projet «Pénélope » publié à cette époque par la Commission européenne et inspiré par François Lamoureux, davantage baigné de la notion traditionnelle d' «exécution » dans le Droit communautaire ${ }^{19}$ et facilitait sans doute l'acceptation ultérieure de la nouvelle catégorie des « règlements délégués » dans les débats de la Convention européenne. ${ }^{20}$

\subsubsection{La gestation de l'article I-36 du Traité constitutionnel}

La Convention, en session plénière, entamait des débats sur le rapport du Groupe IX, dans ses réunions des 5 et 6 décembre 2002, c'est à dire, un peu avant le vote de

\footnotetext{
${ }^{16}$ Cf. www.kangaroogrup.eu/Documents. Dans la tradition du Parlement européen, qui se reflète dans l'article 32 actuel de son Règlement, un intergroupe est une association non-officielle de députés "pour entretenir des échanges de points de vue informels sur des questions spécifiques entre les différents groupes politiques, en ayant recours aux membres des différentes commissions parlementaires, et pour promouvoir les contacts entre les députés et la société civile».

${ }^{17}$ Newsletter, $\mathrm{n}^{\mathrm{O}} 32$, novembre 2002.

${ }^{18} \mathrm{Cf}$. Décision du Parlement européen sur la typologie des actes et la hiérarchie des normes dans l'Union européenne du 17 décembre 2002, Journal Officiel C 31 du 5.2.2004, pp. 126-134, notamment le considérant $\mathrm{F}$ et les paragraphes 13 à 15 .

${ }^{19}$ Vid. infra, sub 3.1.2.

${ }^{20}$ La décision précitée du Parlement avait été transmise à la Convention européenne par Íñigo Méndez de Vigo, membre du Præsidium, le 29 janvier 2003.
} 
la résolution sur le rapport Bourlanges au Parlement européen. En ce qui concerne plus particulièrement la nouvelle catégorie des actes «délégués », le Secrétariat de la Convention était parvenue à la conclusion qu'il y avait eu des avis partagés : certains Conventionnels avaient soutenu le projet du Groupe tandis que d'autres, plus circonspects, avaient demandé plus de précisions avant de se prononcer. ${ }^{21}$ Dans ces conditions, le Président Valéry Giscard d'Estaing avait conclu, en substance, que bien qu'il ne voyait pas encore de consensus sur cette question, il se proposait néanmoins de présenter des propositions plus détaillées sur celle-ci dans l'avenir. ${ }^{22}$

Ces propositions arrivèrent au mois de février 2003, dans le cadre du projet des articles 24 à 33 du Traité constitutionnel présenté par le Præsidium de la Convention, dont l'article 27 était entièrement consacré aux « règlements délégués ». ${ }^{23}$

Le projet des articles sur les instruments juridiques de l'Union a été débattu, pour la première fois, par la Convention européenne, lors de sa session plénière des 17 et 18 mars 2003. Ce projet a reçu un accueil enthousiaste de membres éminents du Parti Populaire Européen et du Parti Socialiste Européen (les deux familles politiques principales du moment); seuls certains « eurosceptiques » ont émis des réticences. ${ }^{24}$ En ce qui concerne l'article 27, il a fait l'objet d'un « large consensus », « de nombreux Conventionnels ayant souligné [le] besoin » d'introduire cette nouvelle catégorie d'actes juridiques. ${ }^{25}$

Le projet a alors été soumis à une procédure de dépôt d'amendements, qui, quant aux « règlements délégués », a donné lieu à des propositions non seulement sur la définition elle-même et la nature juridique de tels actes, mais aussi sur les conditions d'application de ceux-ci et les procédures de contrôle $;{ }^{26}$ certains Conventionnels en ont aussi profité pour livrer une dernière bataille contre leur inclusion dans les actes

${ }^{21}$ Cf. CONV 449/02 du 13 décembre 2002, p. 5.

${ }^{22}$ Ubi supra.

23 « Article 27 : Les règlements délégués

1. Les lois et les lois-cadres européennes peuvent déléguer à la Commission le pouvoir d'édicter des règlements délégués qui complètent ou qui modifient certains éléments non essentiels de la loi ou la loi-cadre.

Les lois et les lois-cadres délimitent explicitement les objectifs, le contenu, la portée et la durée de la délégation. Les éléments essentiels d'un domaine ne peuvent pas faire l'objet d'une délégation. Ils sont réservés à la loi ou à la loi-cadre.

2. La loi ou la loi-cadre détermine explicitement les conditions d'application auxquelles la délégation est soumise qui consistent en une ou plusieurs des possibilités suivantes :

- le Parlement européen et le Conseil peuvent décider de révoquer la délégation,

- le règlement délégué ne peut entrer en vigueur que si, dans le délai fixé par la loi ou la loi-cadre, le Parlement européen ou le Conseil n'expriment pas d'objections,

- les dispositions du règlement délégué sont caduques après un délai déterminé par la loi ou la loicadre. Leur validité peut être prorogée sur proposition de la Commission, par décision du Parlement européen et du Conseil.

Aux fins de l'alinéa précédent, le Parlement européen statue à la majorité de membres qui le composent et le Conseil statue à la majorité qualifiée » (CONV 571/03 du 26 février 2003, p. 7).

${ }^{24}$ Cf. Norman [42], p. 204.

${ }^{25}$ Cf. CONV 630/03 du 21 mars 2003, pp. 2, 3 et 4.

${ }^{26}$ Voir l'analyse du Secrétariat de la Convention, CONV 609/1/03 du 28.3.2003, pp. 11, 12. 
juridiques de l'Union. ${ }^{27}$ Avaient alors suivi la proposition d'un texte révisé et une deuxième série d'amendements visant essentiellement à insister sur les positions déjà bien connues de leurs auteurs. ${ }^{28}$

Au terme de ce processus d'amendements et de révision, l'article 27 original (devenu l'article I-35) a gardé sa structure et son sens général, mais a subi aussi quelques changements importants, notamment dans son paragraphe 2 relatif aux « conditions d'application » auxquelles peut être soumise la délégation.

Tout d'abord, la version revue de cet article, proposée en juin $2003,{ }^{29}$ ne dit plus que les conditions d'application «consistent» en une ou plusieurs des possibilités visées à ce même article, mais qu'elles «peuvent consister» dans une ou plusieurs desdites possibilités, ce qui, à première vue, donnait satisfaction aux Conventionnels qui avaient exprimé leur préférence pour un système plus ouvert, avec une énumération de possibilités indicative et non pas exhaustive.

Le deuxième changement concernait la possibilité de révoquer la délégation : là où il était dit que « le Parlement européen et le Conseil peuvent décider de révoquer la délégation », la version révisée remplace la conjonction «et» par «ou », ce qui résulte sans nul doute de l'insistance de certains Conventionnels qui souhaitaient que la révocation puisse être décidée de manière unilatérale par chacune des deux branches du pouvoir législatif de l'Union, sans que le Parlement européen n'ait à obtenir l'accord du Conseil.

Le troisième changement est le plus évident : la disparition de la troisième possibilité visée à l'alinéa 2, relative à la caducité des actes délégués après un délai déterminé (sunset clause), combattu par certains Conventionnels. ${ }^{30}$

La version de juin 2003 ne subirait ultérieurement aucune autre modification au sein de la Convention européenne ${ }^{31}$ et passa avec succès le rigoureux examen du texte adopté par la Convention que la Conférence intergouvernementale avait confié à un large Groupe d'experts juridiques. ${ }^{32}$ En effet, sur ce point, le Groupe s'était limité à apporter de légères corrections de présentation, visant essentiellement à garantir la cohérence de la terminologie avec celle utilisée dans les autres dispositions et qui n'a pas changé la substance du texte ou apporté plus de clarté à ce dernier. ${ }^{33}$

\footnotetext{
${ }^{27}$ Amendements de David Heathcoat-Amory, de Tunne Kelam, de Liia Hänni et d'Ülo Tärno, de Timothy Kirkhope et du Comte de Stockton.

${ }^{28}$ Cf. CONV 779/03 du 4 juin 2003, pp. 14-15.

${ }^{29}$ Version révisée de la Partie I, CONV 797/03 du 10 juin 2003, p. 29.

${ }^{30}$ Vid. infra 3.2.1.

${ }^{31}$ Cf. CONV 811/03, p. 5

${ }^{32}$ Le Groupe mentionné dans le texte a été présidé par le Jurisconsulte du Conseil et composé d'experts désignés par les États membres et par les États se trouvant alors en cours d'adhésion, par des observateurs des États candidats et par des fonctionnaires des Services juridiques du Parlement, du Conseil et de la Commission : cf. Piris [48], pp. 50-55.

${ }^{33} \mathrm{Il}$ a été affirmé que le groupe d'experts « avait exprimé son désaccord quant à la rédaction formelle de l'article portant sur les actes non législatifs... et avait suggéré une rédaction plus simple et plus claire (CIG 04/03) » et que «la Conférence avait simplifié davantage et ordonné ces articles confus issus de la Convention (CIG50/03)», dans Mangas Martín [34], p. 15 note 29. Le présent auteur a été membre titulaire du Groupe d'experts, mais il ne considère pas que la contribution de ce Groupe ait été si méritoire, en ce qui concerne l'article I-36 : il suffit d'une simple comparaison des textes approuvés par la Convention et
} 
2.2 La délégation de pouvoirs normatifs dans la pratique du Conseil et dans la jurisprudence de la Cour de Justice postérieures

Dans les sombres années ayant suivi les référendums sur la ratification du Traité constitutionnel en France et aux Pays-Bas, l'Union européenne manquait toujours d'une base juridique solide et équilibrée pour la délégation de pouvoirs normatifs. On a alors essayé d'y remédier en ayant recours à des formules qui avaient déjà fait leurs preuves, avec quelques correctifs qui visaient à éviter l'opposition du Parlement européen au cours des procédures de codécision. Par ailleurs, au cours de cette même période, le Parlement a recherché la protection de la Cour de justice face à certaines pratiques abusives du Conseil en matière de «bases juridiques dérivées », ce qui a donné à la Cour l'occasion d'une nouvelle jurisprudence venant éclaircir les difficultés que présentaient ces vieilles pratiques, justement en raison des délégation législatives de facto qu'elles comportaient.

\subsubsection{La délégation dans la Décision 2006/512/CE dans le domaine de la «comitologie»}

En 2006, le Conseil a modifié la Décision 1999/468/CE fixant les modalités de l'exercice des compétences d'exécution conférées à la Commission (plus connue sous le nom de décision sur la «comitologie »), ${ }^{34}$ poussé par la Commission et dans le but de satisfaire, ne serait-ce qu'en partie, les aspirations du Parlement européen. Même si la comitologie a déjà fait l'objet de nombreuses études, ${ }^{35}$ il convient d'apporter quelques précisions afin d'éviter tout malentendu quant aux rapports entre les procédures de comité et les actes délégués.

L'établissement de comités composés de représentants des États membres pour assister (et contrôler) la Commission dans l'adoption de mesures destinées à appliquer des dispositions d'actes législatifs n'est pas une réalité «naturelle » inhérente au système et consacrée depuis les débuts des Communautés européennes. En réalité, dans leur rédaction originale, les Traités n'en faisaient même pas mention. L'article $155 \mathrm{du}$ Traité de la Communauté économique européenne, consacré aux missions de la Commission, prévoyait simplement que cette dernière devait exercer «les compétences que le Conseil lui confère pour l'exécution des règles qu'il établit ». L'apparition des comités était due à des considérations d'ordre pratique (décharger le Conseil, en principe compétent en matière d'exécution, notamment dans le domaine de la Politique Agricole Commune). ${ }^{36}$

L'Acte Unique Européen a consacré cette pratique et reconnu au Conseil la faculté de soumettre l'exercice des compétences d'exécution «à certaines conditions », en

\footnotetext{
par la Conférence pour se rendre compte de la portée strictement formelle des corrections apportées par le Groupe, sans importance pour l'interprète.

${ }^{34}$ Décision 2006/512/CE du 17 juillet 2006, Journal Officiel L 200 du 22.7.2006, p. 11.

${ }^{35}$ Cf. e.g. : Bergström [8] ; Joerges [27] ; Moreiro González [38], pp. 895-923.

${ }^{36}$ Cf. Szapiro [55], pp. 545-586.
} 
ajoutant, en outre, une nouvelle base juridique pour lui permettre de fixer « les principes et les règles » auxquels elles devaient répondre. ${ }^{37}$ Mais ce changement n'avait pas pour objet d'altérer l'équilibre institutionnel : il était nécessaire pour «permettre aux institutions d'exercer leurs compétences dans les conditions les plus conformes à l'intérêt communautaire » ${ }^{38}$ et en finir avec la diversité des procédures de comité, devenues une véritable selva selvaggia. Cela était confirmé par une déclaration de la Conférence Intergouvernementale, dans laquelle cette dernière invitait le Conseil à réserver « une place prépondérante » à la procédure du Comité Consultatif, sans doute la moins exigeante pour la Commission, qui fut suivie de faits.

La « comitologie » a généreusement été utilisée dans la préparation de règles juridiques, en application d'une jurisprudence de la Cour de Justice qui, retenant une notion large d' « exécution », légitimait la délégation de compétences à la Commission pour prendre non seulement des décisions individuelles, mais aussi des mesures de portée générale, pourvu qu'elles ne concernent pas les «éléments essentiels » de l'acte de délégation. ${ }^{39}$

Héritée d'une époque où le Conseil était pratiquement le seul législateur communautaire (le Parlement européen avait alors un rôle purement consultatif et ses avis étaient souvent ignorés), cette situation est devenue une source de difficultés croissantes avec l'apparition de la codécision dans le Traité de Maastricht et l'étendue ultérieure de son champ d'application ratione materiae après le Traité d'Amsterdam. À l'origine de ces difficultés il y avait une incohérence : d'une part, le Parlement européen se dessinait comme l'une des deux branches de l'autorité législative, coresponsable des actes législatifs adoptés par la procédure de codécision avec le Conseil ; mais, d'autre part, le Parlement restait à l'écart du contrôle des mesures d'exécution qui, en outre, restaient soumises aux règles générales fixées par le Conseil, de manière unilatérale. ${ }^{40}$

Le malaise du Parlement européen face à cette anomalie ne l'a pas poussé à s'opposer au recours à la délégation, ${ }^{41}$ mais à le limiter et à rechercher la manière d'exercer son influence. En attestent la Décision de 1999 portant modification du régime de la comitologie, ${ }^{42}$ mais aussi l'ensemble de textes concernant la «procédure Lam-

\footnotetext{
${ }^{37}$ Article 145 du Traité CEE, troisième tiret. Auparavant, l'article 153 chargeait le Conseil d'adopter le statut des «comités visés au Traité »; mais ces comités n'étaient donc pas ceux prévus par le législateur dans le cadre de la «comitologie » : il s'agissait de certains organes directement créés par les Traités qui, par ailleurs, n'étaient pas toujours exclusivement formés de représentants des États membres (ainsi, le Comité du fonds social européen, prévu à l'article $124 \mathrm{CEE}$, avait une composition tripartite).

${ }^{38}$ Préambule de l'Acte unique européen, avant-dernier alinéa.

${ }^{39}$ Arrêt du 17 décembre 1970, Köster, 25/70, Rec. p. 1161, paragraphes 9, 10 ; Recueil, p. 1161.

${ }^{40}$ Les facteurs indiqués dans le texte n'ont pas toujours été pris en compte par la doctrine juridique, avec les perplexités et incompréhensions qui s'en suivent : voir, par exemple, Jiménez Asenjo [26], pp. 265-325 ; notamment pp. 320-322 et 324.

${ }^{41}$ L'affirmation contenue dans le texte a été démontrée empiriquement dans le domaine de l'environnement : cf. Héritier et Moury [23], passim.

${ }^{42}$ Décision 1999/468/CE, Journal Officiel L 184 du 17.7.1999, p. 23. Voir également les déclarations publiées au Journal Officiel C 203 du 17.7.1999, p. 1.
} 
falussy » appliquée à la législation relative aux services financiers, ${ }^{43}$ ou l'Accord interinstitutionnel « Mieux légiférer » de $2003{ }^{44}$

Cependant, la tension interinstitutionnelle sur la délégation n'avait cessé d'augmenter, jusqu'à l'arrivée des propositions «Bâle II », dont le traitement était venu coïncider avec le résultat négatif des deux référendums sur le Traité constitutionnel, ce qui a provoqué une crise et directement conduit à de nouvelles négociations entre les Institutions. ${ }^{45}$

La Décision 2006/512/CE, fruit de ces négociations, reprenait en partie les éléments de l'article I-36 du Traité constitutionnel, avec néanmoins des différences importantes, puisqu'elle restait fidèle à la notion traditionnelle des mesures d' " exécution » et, par conséquent, à l'approche de l'intervention de comités composés de représentants des États membres. L'aspect central de cette réforme, prévu à l'article 2 , tenait en l'ajout d'une procédure, obligatoire dans tous les cas où un acte législatif adopté en codécision par le Parlement européen et par le Conseil prévoyait de déléguer la prise de mesures de portée générale, dite « procédure de réglementation sous contrôle » $(\mathrm{PRAC}) .{ }^{46}$ Cette nouvelle procédure donnait au Parlement européen un véritable droit d'opposition, en toute indépendance de l'avis du comité de réglementation, lui permettant d'empêcher l'adoption de la mesure envisagée par la Commission ou d'entraîner l'abrogation de l'acte délégué, s'il avait déjà été adopté pour des raisons d'urgence. ${ }^{47}$ De plus, il était prévu d'étendre l'application de cette procédure aux actes qui avaient déjà été adoptés en codécision, en remplacement des procédures qui y étaient visées pour l'exécution de leurs dispositions. ${ }^{48}$

Cependant, dans la PRAC, le Parlement devait justifier son opposition en indiquant que le projet de mesures présenté par la Commission «excède les compétences d'exécution prévues dans cet acte de base, ou que ce projet n'est pas compatible avec le but ou le contenu de cet acte, ou qu'il ne respecte pas les principes de subsidiarité ou de proportionnalité $»,{ }^{49}$ alors que le Conseil n'était pas soumis à cette restriction. ${ }^{50}$ Cette asymétrie nous aide à comprendre l'intérêt actuel du Parlement à adapter l'acquis aux règles de l'article 290 du Traité de fonctionnement. ${ }^{51}$

\footnotetext{
${ }^{43}$ Cf. Déclaration du président PRODI devant le Parlement européen du 5 février 2002, Journal Officiel C 284 E du 21.11.2002, p. 19; Lettre du Commissaire Bolkenstein du 2 octobre 2001, ibidem, p. 83; Décision du Parlement européen du 5 février 2002, ibidem p. 115.

${ }^{44}$ Journal Officiel C 321 du 31.12.2003, p. 1.

${ }^{45}$ L'importance de cet épisode a été soulignée par Neergard [40], pp. 129-143.

${ }^{46}$ Cette procédure permettait de «modifier des éléments non essentiels [de l'acte de base], y compris en supprimant certains de ces éléments ou en complétant ledit acte par l'ajout de nouveaux éléments non essentiels » (article 2, nouvel alinéa 2 ajouté par le Règlement 2006/512/CE).

${ }^{47}$ Article 5 bis, paragraphes 3 b), 4 e) et 6 c).

${ }^{48}$ Déclaration trilatérale du 6 juillet 2006, Journal Officiel C 255 du 21 octobre 2006, p. 1.

${ }^{49}$ Décision 2006/512/CE cit. supra, note 34, art. 5 bis, paragraphes 3 b), 4 e) et 6 c).

${ }^{50}$ Cf. Bradley [9], pp. 286-301.

${ }^{51} \mathrm{Cf}$. Résolution du Parlement Européen du 5 mai 2010 sur le pouvoir de délégation législative (rapport Szájer), paragraphe 18. Voir aussi infra, 3.1.1.
} 


\subsubsection{La délégation dans la jurisprudence de la Cour de justice relative aux bases juridiques dérivées}

Dans les années où il était presque le seul législateur de la Communauté européenne, le Conseil, inspiré par des considérations de rapidité et d'efficacité et avec l'aide de la Commission, a développé une longue pratique de «bases juridiques dérivées », ${ }^{52}$ qui permettaient souvent d'éviter ou d'atténuer l'implication du Parlement prévue dans la base juridique applicable du Traité. Cette pratique s'est poursuivie sans grande difficulté malgré l'évolution générale du cadre juridique en faveur d'une plus grande démocratie dans les processus décisionnels des Communautés européennes, notamment par une intervention plus systématique et décisive du Parlement européen, mais a fini par venir se heurter à l'opposition du Parlement, avec deux recours successifs devant la Cour de justice.

Le premier de ces recours a été présenté en 2000, afin de contester un acte pris dans un domaine particulièrement délicat, l'étiquetage de la viande bovine, étroitement lié à la crise de l'Encéphalopathie Spongiforme Bovine (ESB), plus connue sous le nom de «maladie de la vache folle », dans laquelle le rôle du Parlement avait été universellement reconnu, notamment grâce au succès de la commission temporaire d'enquête dont le député Medina Ortega avait été le rapporteur. L'acte litigieux était le Règlement $n^{0}$ 2772/1999 concernant l'étiquetage de la viande bovine, ${ }^{53}$ fondé sur une «base juridique dérivée », l'article 19 du Règlement n ${ }^{\circ} 820 / 97,{ }^{54}$ que le Conseil avait pris pour faire face à la déstabilisation des marchés de produits bovins suite à la crise de l'ESB.

Les faits de cette affaire sont relativement simples. En résumé, le Conseil, confronté à une proposition tardive de la Commission pour proroger la validité des dispositions du Règlement 820/97 relatives à l'étiquetage de la viande bovine et des produits à base de viande bovine (qui expiraient le 31 décembre 1999), avait décidé, pour répondre aux souhaits de la Commission elle-même, que dans le cas où, en première lecture, le Parlement n'accepterait pas la proposition de la Commission sans aucun amendement ou presque, alors il statuerait, non pas eu égard aux dispositions pertinentes de l'article $251 \mathrm{du}$ Traité CE - qui lui imposait la codécision avec le Parlement européen -, mais en application de la procédure prévue à l'article $19 \mathrm{du}$ Règlement 820/97, qui ne prévoyait pas l'implication du Parlement, procédure qui fut effectivement appliquée face au refus du Parlement de s'incliner devant l'ultimatum du Conseil.

Cette affaire n'aurait constitué qu'un cas de conflit de plus sur la base juridique applicable si le Parlement n'en avait pas profité pour attaquer frontalement la thèse soutenue par le Conseil de la «base juridique dérivée », en rejetant l'hypothèse que

\footnotetext{
${ }^{52}$ Les « bases juridiques dérivées » ont été définies comme suit : « Nella prassi istituzionale con l'espressione "base giuridica derivata" si intende la tecnica legislativa che distingue due piani normativi derivati. Nell'atto di base si introduce un fondamento normativo che, nel contemplare un meccanismo de produzione giuridica di secondo grado, disciplina la formazione della volontà delle istituzioni secondo modalità non coincidenti con quelle previste dai Trattati ». Baratta [6], pp. 2076-2079; p. 2076.

53 Journal Officiel L 334 du 28 décembre 2009, p. 1.

${ }^{54}$ Journal Officiel L 117 du 7 mai 1997, p. 1.
} 
les Traités permettraient d'adopter, outre des actes normatifs et des actes d'exécution, une catégorie d' «actes d'un troisième type » fondés sur cette classe de bases juridiques. ${ }^{55}$ Cependant, la Cour a décidé de se limiter à annuler le Règlement attaqué compte tenu qu'en tout cas, la modification de son champ d'application dans le temps «ne pouvait intervenir que sur le fondement d'une base juridique de nature équivalente à celle sur le fondement de laquelle le règlement lui-même avait été adopté », ${ }^{56}$ en indiquant expressément qu'il n'était dès lors pas nécessaire de se prononcer sur les autres moyens du Parlement. ${ }^{57}$

Au moment où il a été rendu, cet arrêt est passé presque inaperçu dans la doctrine juridique ${ }^{58}$ et il a ensuite été critiqué, justement pour ne pas avoir examiné la question des bases juridiques dérivées, même s'il était tout de même significatif que la Cour n'eut pas fait grand cas des arguments soulevés par le Conseil et par la Commission sur ce point, qui trouvaient le soutien de l'Espagne ${ }^{59}$ et des conclusions de l'Avocat Général Mme Stix-Hackl.

L'arrêt rendu dans l'affaire C-93/00 était intervenu presque en même temps que l'adoption de la Déclaration de Laeken par le Conseil européen. Or, en 2006, année à laquelle le Parlement présentait son deuxième recours, la situation était bien différente : à cette époque, le texte du Traité constitutionnel sur les «règlements délégués » était déjà connu, et il prévoyait que de tels règlements pourraient être adoptés seulement par la Commission (et non pas par le Conseil) et uniquement dans les conditions prévues dans le Traité lui-même.

L'objet du recours était l'annulation des articles 26, paragraphes 1 et 2, et 36, paragraphe 3, de la Directive 2005/85 du Conseil, relative aux normes minimales que les États membres doivent appliquer pour l'octroi et le retrait du statut de réfugié. ${ }^{60}$ Ces dispositions visaient un aspect d'un intérêt évident, celui de la détermination des pays «sûrs » aux fins d'évaluer si les demandes d'asile sont ou ne sont pas justifiées du point de vue de la sécurité du demandeur, et prévoyaient l'établissement de listes de pays sûrs au travers d'une procédure simplifiée qui, sans aller jusqu'à exclure la participation du Parlement européen, l'allégeait en remplaçant la procédure de codécision, en principe applicable, par une simple consultation non contraignante.

Comme le dirait l'Avocat général M. Poiares Maduro dans ses conclusions, « la question centrale de [cette] affaire, qui a justifié son renvoi devant la grande chambre [est] celle de la possibilité pour le législateur communautaire de recourir à des bases juridiques dérivées ». ${ }^{61}$ Or, sur ce problème général, les positions des parties étaient clairement opposées : alors que le Parlement soulignait que les Traités n'avaient pas

\footnotetext{
${ }^{55}$ Arrêt du 13 décembre 2001, Parlement/Conseil, C-93/00, Rec. p. I-10119, paragraphes 23 et 24.

${ }^{56}$ Loc. cit., paragraphes $42-44$.

${ }^{57}$ Loc. cit., paragraphe 45.

${ }^{58}$ Voir, cependant, l'exception notable de Vedaschi [56], pp. 903-908.

${ }^{59}$ L'Espagne a été le seul État membre qui a cru utile d'intervenir au soutien des conclusions du Conseil dans cette affaire.

${ }^{60}$ Journal Officiel L 326 du 13 décembre 2005, p. 13.

${ }^{61}$ Conclusions de l'Avocat général M. Poiares Maduro du 27 septembre 2007, Parlement européen/Conseil, C-133/06, paragraphe 9. Dans ces conclusions, cette question est étroitement liée à la délégation de pouvoirs normatifs, avec des références au droit comparé pertinent.
} 
prévu l'adoption de bases juridiques dérivées et que la Commission le soutenait en rappelant que les Institutions ne peuvent agir que dans la limite des compétences qui leur ont été attribuées, le Conseil, soutenu par la France, affirmait que rien ne s'opposait à l'adoption de bases juridiques dérivées et insistait sur le fait qu'en cette matière, il était conforté par une pratique consolidée. ${ }^{62}$

La Cour de justice a statué en faveur du Parlement et a annulé les dispositions attaquées, considérant essentiellement que « reconnaître à une institution la possibilité d'établir des bases juridiques dérivées, que ce soit dans le sens d'un renforcement ou dans celui d'un allégement des modalités d'adoption d'un acte, reviendrait à lui attribuer un pouvoir législatif qui excède ce qui est prévu par le Traité »; et que « cela conduirait également à lui permettre de porter atteinte au principe de l'équilibre institutionnel, qui implique que chacune des institutions exerce ses compétences dans le respect de celles des autres ». ${ }^{63}$

Il s'agit d'une jurisprudence porteuse de nouveaux éléments puisque, même s'il est vrai que la Cour avait déjà déclaré que « les règles relatives à la formation de la volonté des institutions communautaires sont établies par le Traité et elles ne sont donc à la disposition ni des États membres ni des institutions elles-mêmes », ${ }^{64}$ il n'en demeure pas moins qu'elle l'avait fait dans un contexte différent, celui de la nature impérative des majorités prévues dans les Traités pour la formation de la volonté des Institutions. De plus, la Cour ne pouvait ignorer qu'avec cet arrêt, elle allait mettre fin à une longue pratique, que le Conseil semblait considérer comme un élément faisant partie intégrante de l'acquis communautaire. ${ }^{65}$

\section{Les actes délégués dans le Traité de Lisbonne}

L'article 290 du Traité sur le fonctionnement de l'Union européenne (TFUE) reprend presque littéralement le texte de l'article I-36 du Traité constitutionnel, ${ }^{66}$ ce qui ne semble pas être le résultat d'une simple inertie compte tenu du mandat détaillé que la Conférence Intergouvernementale avait reçu du Conseil européen des 21 et 22 juin 2007 et de l'examen scrupuleux auquel les textes ont été soumis au sein d'un nouveau Groupe d'experts juridiques nommés par les Institutions et par les États membres. ${ }^{67}$ Les seules différences sont un reflet du changement de terminologie pour les actes législatifs - le retour aux traditionnels « règlements » et «directives », à la place

\footnotetext{
${ }^{62}$ Arrêt du 6 mai 2008, Parlement/Conseil, affaire C-133/06, notamment les paragraphes 24, 28, 31 et 40.

${ }^{63}$ Loc. cit., paragraphes 56 et 57 .

${ }^{64}$ Arrêt du 23 février 1988, Royaume-Uni/Conseil, affaire 68/86, Rec. p. 855, paragraphe 38.

65 À la pratique du Conseil de prévoir des bases juridiques dérivées, il faut ajouter ses «autodélégations » de la fonction exécutive, par ailleurs reconnues par la jurisprudence, bien qu'avec d'importantes restrictions. Dans ces conditions, il ne semble pas facile de partager le constat d'après lequel « le mécanisme créé par le Conseil dans cette affaire, en vertu duquel une institution se fait une délégation à elle-même est innovateur, ce qui ne le rend pas moins insensé », dans Navarro Batista [39], p. 12.

${ }^{66}$ Les références à des textes du Traité de Lisbonne ou des protocoles et déclarations s'y rapportant ont été tirées de la version révisée des versions consolidées des Traités, publiée au Journal Officiel C 83 du 30 mars 2010, avec la Charte des droits fondamentaux.
}

${ }^{67}$ Cf. Pennera [45], pp. 7-24. 
des «lois » et des «lois-cadres » du Traité constitutionnel - qui a rendu presque incontournable, pour éviter toute confusion, de remplacer «règlements délégués », par «actes non législatifs de portée générale» ou «actes délégués » dans le texte du Traité ; cela a également conduit à l'ajout d'un nouvel alinéa 3, aux termes duquel «l'adjectif « délégué » ou « déléguée » est inséré dans l'intitulé des actes délégués », ce qui, en passant, venait confirmer la possibilité pour la Commission d'adopter des actes délégués du type «directive».

Cette identité presque totale des textes relatifs aux actes délégués dans le non né Traité constitutionnel et dans le TFUE actuellement en vigueur ${ }^{68}$ confirme l'intérêt actuel de la doctrine publiée à propos du premier de ces Traités pour l'interprétation et l'application du deuxième, bien qu'avec certaines précautions résultant aussi bien de l'intention évidente de «déconstitutionnalisation » ayant été à l'origine du passage de l'un à l'autre, ${ }^{69}$ que des modifications dans le contexte général du Traité de Lisbonne et de ses Protocoles, qui ont fait naître de nouvelles questions.

\subsection{Le pourvoir de délégation et la préparation des actes délégués}

L'article 290 TFUE permet au législateur de déléguer le pouvoir d'adopter des dispositions de portée générale, sans avoir à adopter, au préalable, un acte réglementant de manière générale l'exercice de ce pouvoir de délégation, à la différence du régime prévu à l'article 291 pour les mesures d'exécution, porteur d'une base juridique pour l'établissement de «normes et de principes généraux », dans la tradition de la comitologie ultérieure à l'Acte unique européen.

À l'occasion de l'entrée en vigueur du Traité de Lisbonne, la Commission européenne a annoncé la ligne de conduite qu'elle envisageait de suivre pour la mise en œuvre de l'article 290 dans l'exercice de son droit d'initiative ou des compétences qui lui seraient déléguées. ${ }^{70}$ Cette démarche a permis au Parlement européen ${ }^{71}$ et au Conseil $^{72}$ de prendre position et a favorisé le développement d'une pratique presque uniforme par le recours à des «modèles » utilisés sans grandes variations dans les actes législatifs, sans préjudice de la liberté juridique du législateur. ${ }^{73}$

\footnotetext{
${ }^{68}$ L'absence de modifications substantielles mentionnée reste fidèle à l'approche de continuité du cadre général des dispositions du Traité de Lisbonne sur le droit dérivé, déjà observé dans Balaguer Callejón [4], p. $65 ;$ p. 72.

${ }^{69}$ Il semble peu vraisemblable que le Traité de Lisbonne présente les mêmes potentialités qui, tout au moins, ne pouvaient être exclues dans le cas du Traité constitutionnel, sur lesquelles cf. Jacqué [25], pp. $41-52$.

${ }^{70}$ Communication de la Commission au Parlement européen et au Conseil - Mise en æuvre de l'article 290 du Traité sur le fonctionnement de l'Union européenne, COM (2009) 673 final, Bruxelles, 9.12.2009.

${ }^{71}$ Cf. Résolution du Parlement européen du 5 mai 2010 sur les pouvoirs de délégation législative (rapport Sczajer).

${ }^{72}$ Cf. Rapport de la présidence au COREPER sur les actes délégués, Doc. 16998/09 du 2 décembre 2009.

${ }^{73}$ Le Parlement européen avait expressément proposé le recours à de tels «modèles » : cf. Résolution du 7 mai 2009 sur les nouvelles compétences du Parlement européen et ses responsabilités dans la mise en œuvre du Traité de Lisbonne, point 68.
} 


\subsubsection{Le pouvoir de délégation : contenu et portée}

L'article 290 TFUE, dans son paragraphe $1^{\mathrm{er}}$, dispose de ce qui suit :

«Un acte législatif peut déléguer à la Commission le pouvoir d'adopter des actes non législatifs de portée générale qui complètent ou modifient certains éléments non essentiels de l'acte législatif.

Les actes législatifs délimitent explicitement les objectifs, le contenu, la portée et la durée de la délégation de pouvoir. Les éléments essentiels d'un domaine sont réservés à l'acte législatif et ne peuvent donc pas faire l'objet d'une délégation de pouvoir.»

Cette disposition s'inspire des traditions constitutionnelles des États membres sur la délégation législative, mais diffère d'elles dans des aspects significatifs, ce qui a été à l'origine de certaines incompréhensions à propos de la rédaction presque identique du Traité constitutionnel, qu'il convient à présent de surmonter.

L'article 290 reconnaît au législateur un pouvoir et en règle l'exercice, mais il ne l'oblige pas à l'utiliser, ce qu'a rappelé la Commission elle-même en disant qu' «il est loisible au législateur de réglementer de façon pleine et entière un domaine d'action $\gg{ }^{74}$

Le champ d'application du pouvoir de délégation n'est soumis à aucune limite, que ce soit en raison du type d'acte législatif ou en raison de la procédure législative qui a été suivie pour l'adoption de cet acte. Tout d'abord, rien dans le texte de l'article 290 ne permet d'exclure les directives de son champ d'application : les avertissements de certains auteurs, quant aux difficultés de la délégation du pouvoir législatif dans le cas des lois-cadres prévues dans le Traité constitutionnel ${ }^{75}$ n'ont pas été entendus, probablement parce que limiter la délégation aux seuls règlements aurait trop limité l'intérêt du pouvoir de déléguer, mais aussi parce que la mise en œuvre de la Décision 2006/512/CE n'avait pas confirmé l'existence de difficultés dans de pareils cas. ${ }^{76}$ Deuxièmement, le pouvoir de délégation ne semble pas être limité - comme c'était le cas dans la PRAC - à la « procédure législative ordinaire » (la codécision du Parlement européen et du Conseil) $i^{.77}$ en d'autres termes, cette faculté peut tout aussi bien être exercée dans le cadre des procédures législatives « spéciales ». ${ }^{78}$

Quant aux éventuelles limitations du pouvoir de déléguer ratione materiae, elles ne figurent pas à l'article 290, mais il est clair que leur existence dépend aussi d'autres dispositions des Traités, qui doivent s'appliquer, de préférence, en vertu du principe de spécialité. Par exemple, dans le cadre de l'auto-organisation des Institutions européennes, des dispositions de portée générale non comprises dans leurs Règlements

\footnotetext{
${ }^{74} \mathrm{COM}(2009) 673$ cit., paragraphe 2.3 in fine.

${ }^{75}$ Cf. Balaguer Callejón [3], pp. 61-79; p. 78 ; Vírgala Foruria [57], pp. 279-324 ; p. 305.

${ }^{76}$ Les exemples ne manquent pas, sachant que la procédure de réglementation avec le contrôle ajouté par la Décision 2006/512/CE était obligatoirement applicable à tous les actes adoptés en codécision et qu'en outre, les Institutions s'étaient engagées à l'appliquer à tous les actes antérieurs adoptés au travers de cette procédure, sans distinguer le type d'acte concerné : vid. supra 2.2.1.

${ }^{77}$ COM (2009) 673 final cit., paragraphe 2.

${ }^{78} \mathrm{La}$ position soutenue dans le texte n'est pas expressément retenue dans certaines des premières contributions sur l'article 290 : voir Gutiérrez Espada [22], p. 47.
} 
internes, mais adoptées par des organes de l'Institution sur le fondement de ces Règlements ou sur le fondement de dispositions d'actes législatifs, continueront probablement à exister. ${ }^{79}$

Seuls peuvent faire l'objet de délégation des éléments autres que les éléments « essentiels », puisqu'aux termes du premier paragraphe de l'article 290, la réglementation de ces derniers est clairement et de manière réitérée réservée aux actes législatifs. Or, il est difficile de partager l'avis selon lequel il y a lieu de « laisser au règlement délégué les aspects simplement accessoires ou de détail, en établissant dans la loi, de manière prolixe, tous les éléments constitutifs de la matière $»,{ }^{80}$ car une vision aussi restrictive ne saurait trouver appui au sens ordinaire du terme «essentiel », ni dans la jurisprudence de la Cour de justice sur la délégation de compétences exécutives (qui est à l'origine du recours à cette notion), ni dans les travaux préparatoires de cette disposition. ${ }^{81}$ Par ailleurs, dans la Convention européenne, les amendements visant à introduire des indications sur les éléments qui devraient être considérés comme « essentiels », ou qui auraient contraint le législateur d'en introduire, ont échoué. ${ }^{82}$

La limitation du pouvoir de délégation aux seuls éléments «non essentiels » pose aussi une autre question qui, semble-t-il, n'a pas encore été examinée par les Institutions européennes : la possibilité de recourir à la délégation pour l'adoption de textes refondus, de manière à éviter les difficultés qui se présentent dans le cadre de la mise en œuvre des procédures actuellement prévues à cet effet. ${ }^{83}$

Quant à la délimitation de la délégation, aux termes de l'article 290, l'acte législatif de base « détermine explicitement les objectifs, le contenu, la portée et la durée de la délégation de pouvoir ». La Commission a tiré la conséquence d'après laquelle «la délégation de pouvoir doit être claire, précise et détaillée ». ${ }^{84}$

Les actes délégués peuvent compléter ou modifier des éléments non essentiels de l'acte législatif. La première variante n'a trouvé presque aucune résistance à la Convention européenne, abstraction faire des Conventionnels qui s'opposaient à toute forme de délégation législative $;{ }^{85}$ en revanche, elle a surpris certains auteurs, compte tenu de sa proximité avec le genre de mesures qui pouvaient être adoptées antérieurement à travers les procédures de la «comitologie», sans comprendre que l'article

\footnotetext{
${ }^{79} \mathrm{Il}$ y a des exemples, entre autres, dans les articles 17 et 18 du Statut des Députés, qui confient au Parlement européen la charge de déterminer les conditions d'exercice des droits relatifs à la retraite et à l'assurance maladie de ses membres. Cf. Décision 2005/648/CE, Euratom, Journal Officiel L 262 du 7 octobre 2005, p. 1.

${ }^{80}$ Vírgala Foruria [57], p. 306.

${ }^{81}$ Vid. supra, 2.1.2.

${ }^{82} \mathrm{Cf}$. les amendements à l'article 27 originaire présentés par MM. Tiilikainen, Peltomäki, Kiljunen, Vanhanen, Korhonen et Helle, par M. Queiró et par M. Fini.

${ }^{83} \mathrm{Cf}$. Accord Interinstitutionnel du 26 novembre 2001, pour un recours plus structuré à la technique de la refonte des actes juridiques, Journal Officiel C 77 du 28.03.2002, p. 1; Garzón Clariana [19], pp. 61-64.

${ }^{84} \mathrm{COM}$ (2009) 673 final cit., paragraphe 3.1.

${ }^{85}$ Voir néanmoins l'amendement à l'article I-35 présenté par les Conventionnels Messieurs Lopes et Lobo Antunes.
} 
290 existe justement pour éviter certaines difficultés et incohérences desdites procédures. $^{86}$

En revanche, la possibilité que la Commission fût autorisée à modifier les dispositions de l'acte législatif avait dû lutter contre une opposition considérable de Conventionnels influents de différentes tendances politiques. ${ }^{87} \mathrm{C}$ 'est peut-être pour cette raison que la Commission, dans un geste rassurant, avait montré une préférence pour circonscrire les possibilités de modification de manière très précise, voire de conditionner cette dernière à la production de certains faits ou de la limiter d'une autre façon. ${ }^{88}$ Il convient cependant de se demander si cette tendance sera suivie dans les actes législatifs, car elle restreindrait l'utilité de la délégation sans aucun motif apparent. ${ }^{89}$

Quant à la durée de la délégation, l'exigence que l'acte législatif de délégation délimite explicitement la durée de la délégation a donné lieu à des interprétations dans le sens que la délégation doit être donnée pour une durée déterminée, voire qu'elle doit s'éteindre par l'adoption d'un seul acte délégué. ${ }^{90}$ Mais ces limites, trop attachées à des conceptions propres au Droit étatique, ${ }^{91}$ ne trouvent d'appui ni dans la lettre de l'article 290 ni dans les travaux préparatoires. La Commission a souligné, au contraire, que les délégations de pouvoir normatif « devraient en principe être à durée indéterminée », compte tenu du besoin de "garantir la continuité de l'adoption des actes juridiques essentiels à la mise en œuvre des politiques de l'Union ». ${ }^{92}$ Dans ce même sens, le Parlement européen a lui-même reconnu que «la durée de la délégation peut être indéterminée ${ }^{93}$

Dans la pratique, la mise en œuvre de l'article 290 confirme que le législateur peut toujours opter pour une délégation soit à durée indéterminée, ${ }^{94}$ soit à durée déterminée. ${ }^{95}$ La formule la plus habituelle consiste en la fixation d'une durée de cinq

\footnotetext{
${ }^{86}$ Vid. supra, 2.2.1. Sur la notion de «compléter » et sur la limite avec les actes d'exécution, voir notamment Jacqué [24], pp. 467, 468.

${ }^{87}$ Voir les amendements présentés à l'article 27 par MM. Borrell, Carnero et López Garrido, ainsi que par M. Teufel, et les amendements à l'article I-35 ultérieur présentés par Mme Hjelm-Wallén et MM. Peteson et Lekberg, ou par M. Wuermeling.

${ }^{88}$ Cf. COM(2009)673 final cit., paragraphe 3.1.

${ }^{89}$ Voir, par exemple, la Directive 2010/65 du 20 octobre 2010 concernant les formalités déclaratives applicables aux navires à l'entrée et/ou à la sortie des ports des États membres, Journal Officiel L 283 du 29.10.2010, p. 1 .

${ }^{90}$ Cf. Vírgala Foruria [57], pp. 309-311.

${ }^{91}$ La perception des délégations législatives en Droit constitutionnel est marquée par l'expérience des « délégations en blanc ou lois de pleins pouvoirs » comme celle approuvée en Allemagne en 1933 au bénéfice du chancelier Hitler : cf. e.g. Otto [43], p. 183, 187. Évidemment, les préoccupations de ce genre n'existent pas lorsque, s'inspirant du Droit Administratif, les actes délégués sont assimilés aux règlements, plus qu'aux lois déléguées : cf. Baño Léon [5], p. 7.

${ }^{92}$ COM (2009) 673 cit., paragraphe 3.2.

${ }^{93}$ Résolution du 5 mai 2010 cit., paragraphe 8.

${ }^{94}$ Voir, par exemple, l'article 36 de la Directive 2010/35 du 16 juin 2010 relative aux équipements sous pression transportables, Journal Officiel L 165 de 30.6.2010, p. 1.

${ }^{95}$ La durée de la délégation peut être fixée en nombre d'années, mais elle peut également l'être par l'indication de la date d'extinction de la délégation : voir, par exemple, l'article 12 de la Directive 2010/40
} 
ans avec tacite reconduction, à moins que le Parlement européen ou le Conseil ne décident de révoquer la délégation. ${ }^{96}$

Quant aux effets juridiques de l'acte de délégation, la thèse la plus probable est que ce dernier crée une obligation à charge de la Commission d'exercer les pouvoirs qui lui ont été conférés, obligation semblable à celle qui, en Droit constitutionnel, lie le gouvernement dans les cas de délégation législative. ${ }^{97}$ En revanche, rien ne semble empêcher la Commission, dans l'exercice de son droit d'initiative, de proposer, avant l'arrivée du terme de la délégation, des modifications de l'acte législatif de délégation ayant trait aux termes de la délégation; de même, rien ne semble empêcher le Parlement européen ou le Conseil de demander à la Commission de proposer de telles modifications en application des articles 225 et 241 TFUE. Il convient d'en dire de même de l'initiative citoyenne visée à l'article 11, paragraphe 4, du Traité de l'Union européenne.

\subsubsection{La procédure de préparation des actes délégués}

L'article 290 ne prévoit que la possibilité de déléguer «à la Commission » le pouvoir d'adopter des actes non législatifs de portée générale. Même si la possibilité de déléguer ce pouvoir aussi au Conseil avait initialement été envisagée, ${ }^{98}$ cette idée avait bientôt été abandonnée à la Convention européenne. De même, la proposition de confier ce pouvoir à des Agences indépendantes avait également été rejetée, ${ }^{99}$ probablement parce qu'une fois la délégation conçue comme une procédure allant au-delà de la réglementation des aspects techniques, l'argumentation en faveur de cette possibilité s'en trouvait affaiblie.

Ce monopole de la Commission s'accompagne d'une «autonomie considérable ${ }^{100}$ dans la préparation des actes délégués. ${ }^{101}$ Or, la Commission a pris des engagements d'une importance croissante. Déjà, à la Conférence Intergouvernementale, elle s'était engagée à respecter la procédure suivie dans le domaine des services financiers (la « procédure Lamfalussy ») et donc à consulter des experts désignés par

relative aux systèmes de transport intelligents dans le domaine du transport routier, Journal Officiel L 207 du 6.8.2010, p. 1 ; et l'article 10 du Règlement 911/2010 concernant le Programme européen de surveillance de la Terre (GMES), Journal Officiel L 276 du 20.10.2010, p. 1.

${ }^{96}$ Voir, par exemple, la Directive 2010/45 du 7 juillet 2010 relative aux normes de qualité et de sécurité des organes humains destinés à la transplantation, Journal Officiel L 207 du 6.8.2010, p. 14.

${ }^{97}$ Cf. e.g. Pérez Royo [47], p. 208. La thèse soutenue dans le texte semble être partagée par la Commission puisque, dans les premiers exemples d'actes délégués, la motivation commence par l'idée que l'acte de base «exige de la Commission que cette dernière adopte des actes délégués » (italique ajoutée). Voir, par exemple, le Règlement délégué n ${ }^{0} 1060 / 2010$ du 28 septembre 2010 concernant l'indication, par voie d'étiquetage, de la consommation d'énergie des appareils de réfrigération ménagers, Journal Officiel L 314 du 30.11.2010, p. 17.

${ }^{98} \mathrm{Cf}$. Rapport de synthèse relatif à la session plénière des 5 et 6 décembre 2002, CONV 449/02 du 13 décembre 2002, p. 2.

${ }^{99} \mathrm{Cf}$. Résolution du Parlement européen du 17 décembre 2002 cit., paragraphes 13 et 16.

${ }^{100} \mathrm{COM}$ (2009) 673 final cit., paragraphe 4.2.

${ }^{101}$ Le paragraphe 3 de l'article 290 ne pose qu'une seule condition de forme : vid. supra 3.1 . 
les États membres dans ce domaine. ${ }^{102}$ Mais surtout, après l'entrée en vigueur du Traité de Lisbonne, la Commission a annoncé son intention de consulter de façon systématique les experts des autorités nationales de tous les États membres qui seront chargés de la mise en œuvre des actes délégués et, à cette fin, d'avoir recours aux groupes d'experts déjà existants ou même d'en constituer de nouveaux. ${ }^{103}$

Cette association de groupes d'experts ne saurait être confondue avec la «comitologie » : il n'existera plus de procédure qui permette aux experts de faire obstacle à l'adoption de l'acte délégué ou de renvoyer la question au Conseil. ${ }^{104} \mathrm{D}$ 'autre part, l'assistance des groupes visés par la Commission peut faciliter la mise en œuvre correcte des actes délégués dans les Etats Membres. ${ }^{105}$ Toujours est-il qu'une telle procédure ne correspond pas aux attentes de la Convention européenne. 106

D'un point de vue juridique, rien ne semble empêcher la Commission de rechercher d'autres conseils, par exemple auprès des Agences européennes compétentes en raison de la matière, ou auprès d'institutions scientifiques ou techniques de prestige reconnu. ${ }^{107} \mathrm{La}$ Commission peut ainsi, si elle le souhaite, avoir recours à des «experts techniques alternatifs» dont l'éventuelle utilité avait déjà été signalée par la doctrine dans le cadre de la comitologie. ${ }^{108}$

Le Parlement européen a déploré l'approche de la Commission, considérée comme trop continuiste ${ }^{109}$ et a réagi en insistant sur la nécessité de rester intimement lié à l'élaboration des actes délégués. ${ }^{110}$

Dans la pratique, les actes législatifs qui confèrent à la Commission le pouvoir d'adopter des actes délégués contiennent, dans leur motivation, une phrase d'après laquelle « Il est particulièrement important que la Commission procède aux consultations appropriées tout au long de son travail préparatoire, y compris au niveau des

${ }^{102} \mathrm{Cf}$. Déclaration n ${ }^{\mathrm{O}} 39$ annexée à l'Acte final de la Conférence intergouvernementale, prenant note de l'intention de la Commission. Ce texte se trouvait déjà dans la Déclaration $n^{0} 8$ annexée à l'Acte final de la CIG qui a adopté le Traité constitutionnel et avait alors été considéré comme un élément non dénué d'intérêt aux fins de faciliter l'acceptation du Traité : cf. Duff [17], p. 98.

${ }^{103}$ COM (2009) 673 final cit., paragraphe 4.2. L'assistance des experts visés dans le texte avait été considérée comme indispensable par le Conseil : cf. Doc. 16998 cit., p. 3.

${ }^{104}$ Cette différence a été mal perçue par les observateurs peu intéressés par la situation juridique : cf. Guéguen [21], p. 70. Voir, en revanche, la nette présentation de Ponzano [50], p. 135 ; pp. 136-138.

${ }^{105}$ Cf. Piris [49], p. 103.

${ }^{106}$ La Convention européenne n'avait pas bien accueilli la thèse de certains de ses membres, d'après laquelle il fallait obliger la Commission à se faire assister par des comités ou des groupes d'experts dans le cadre de l'élaboration des actes délégués : les amendements qui avaient été présentés dans ce sens par M. Schlütter, MM. de Vries et Bruijn ou M. Hain n'avaient pas prospéré.

${ }^{107}$ Le conseil mentionné dans le texte ne saurait être confondu avec une cession des fonctions de la Commission, incompatible avec le Droit de l'Union d'après la jurisprudence classique de la Cour de justice faite dans l'affaire Meroni (arrêt du 13 juin 1958, affaire 9/56, Rec. p. 11).

${ }^{108}$ Cf. Neuhold [41], p. 1 ; pp. 12-13.

${ }^{109}$ Exposé des motifs du rapporteur M. Szájer, PE439.171v03-00 du 29.3.2010.

${ }^{110}$ La Résolution du 5 mai 2010 cit, plaide en faveur de la conclusion d'un accord interinstitutionnel prévoyant des « consultations au cours de la préparation et de l'élaboration des actes délégués » et insiste aussi sur le fait que la Commission « doit donner au Parlement un accès aux réunions préparatoires, un échange de points de vue et des consultations se rapportant aux actes délégués concernés » (paragraphe 10 premier tiret et paragraphe 11 deuxième tiret). 
experts ». ${ }^{111}$ Cette formule semble être suffisamment souple pour permettre à la Commission aussi bien de requérir les conseils des techniciens qui conviennent, que d'éviter des éventuelles difficultés dans le cadre du contrôle de l'exercice de son pouvoir délégué par le législateur de l'Union.

\subsection{Le contrôle des délégations et des actes délégués}

L'article 290 TFUE, dans son paragraphe 2, dispose ce qui suit :

«Les actes législatifs fixent explicitement les conditions auxquelles la délégation est soumise, qui peuvent être les suivantes :

a) le Parlement européen ou le Conseil peut décider de révoquer la délégation;

b) l'acte délégué ne peut entrer en vigueur que si, dans le délai fixé par l'acte législatif, le Parlement européen ou le Conseil n'exprime pas d'objections.

Aux fins des points a) et b), le Parlement européen statue à la majorité des membres qui le composent et le Conseil statue à la majorité qualifiée. »

Comme l'a dit la Commission dans sa Communication du mois de décembre 2009, cet article prévoit le pouvoir de contrôle des actes délégués que le Traité reconnaît aux deux branches du pouvoir législatif de l'Union. ${ }^{112}$ Or, il convient d'ajouter que, dans ce cadre, il existe en plus des procédures prévues dans d'autres dispositions des Traités ou des Protocoles, qui doivent aussi être examinées de manière à obtenir une vue d'ensemble de la matière.

\subsubsection{Le contrôle politique du législateur et des Parlements des États membres}

En termes politiques, le contrôle fait partie des «conditions requises de la délégation» sur lesquelles la Convention européenne a porté toute son attention, suite à la préparation soignée de son Groupe de travail IX. ${ }^{113}$ En comparaison, les contributions de la doctrine juridique sur ce point sont restées relativement modestes.

Sur la nécessité du contrôle, il a été suggéré que l'utilisation du verbe « pouvoir » au début du paragraphe 2 de l'article 290 permettait au législateur de déléguer, sans fixer explicitement les modalités du contrôle sur les actes délégués. ${ }^{114}$ Or, non seulement cette interprétation n'est pas la seule possible (comme nous le verrons plus loin), mais elle ne semble ni cohérente avec le contexte ${ }^{115}$ ni compatible avec la ratio

\footnotetext{
${ }^{111}$ Voir, par exemple, le considérant $n^{\circ} 6$ du Règlement 640/2010 du 7 juillet 2010 établissant un programme de documentation des captures de thon rouge, Journal Officiel L 194 du 24.7.2010 p. 1, p. 2.

${ }^{112} \operatorname{COM}(2009) 673$ final cit., paragraphe 5.1.

${ }^{113}$ Cf. Méndez de Vigo [36], p. 272.

${ }^{114}$ Cf. Masson [35], pp. 157-173; p. 169 note 39, et la bibliographie qui y est citée. L'insistance sur le contrôle a surpris, du point de vue du Droit administratif interne, en ce sens que les éventuelles difficultés pratiques devraient être réglées par le rang infra-légal des actes délégués : cf. Baño León [5], pp. 5-6. Cette thèse ne tient compte, entre autres choses, ni des singularités de l'initiative législative dans l'Union européenne ni du fait que dans l'Union, le Parlement n'est pas le seul législateur.

${ }^{115}$ Le paragraphe 2 de l'article 290 commence en disant que « les actes législatifs fixent explicitement les conditions » en utilisant la forme impérative qui garde en outre toute symétrie avec celle employée au paragraphe $1^{\mathrm{er}}$ de ce même article portant sur la délimitation de la délégation de pouvoir.
} 
legis des actes délégués, qui implique que le législateur ne doive être à aucun moment dépossédé de ses prérogatives. ${ }^{116}$

En ce qui concerne les institutions de contrôle, le paragraphe 2 de l'article 290 consacre l'égalité du Parlement européen et du Conseil, ${ }^{117}$ même s'il faut ajouter qu'il y a aussi une asymétrie dans la mesure où, pour déclencher les mécanismes de contrôle, il suffit au Conseil d'obtenir la majorité qualifiée, aujourd'hui généralement applicable à cette Institution, sauf exception, alors que le Parlement requiert la réunion des voix de «la majorité des membres qui le composent», dans la pratique beaucoup plus difficile que la majorité des membres présents et votants, qui s'applique en règle générale. ${ }^{118}$

La parité entre le Parlement européen et le Conseil est ici le reflet de la longue lutte du Parlement en faveur d'une correspondance entre le pouvoir législatif de délégation et le pouvoir de contrôle des actes délégués. ${ }^{119}$ Or, cette correspondance implique aussi le fait que lorsque la délégation est exclusivement attribuable soit au Parlement, soit au Conseil, dans la mise en œuvre d'une procédure législative spéciale, le contrôle incombe alors uniquement à l'Institution auteur de l'acte. ${ }^{120} \mathrm{Ce}$ corolaire semble confirmé par la pratique. ${ }^{121}$

Le premier des modes de contrôle visés à l'article 290 est la révocation (call back). La révocation n'est dirigée contre aucun acte délégué en particulier, mais suppose le retrait, total ou partiel, de la confiance déposée en la Commission, ${ }^{122}$ mettant fin à la délégation, sous réserve des effets des actes délégués déjà adoptés.

Dans le cadre du Traité constitutionnel, il y a eu une discussion sur la question de savoir si la révocation pouvait être décidée unilatéralement par le Parlement européen ou par le Conseil ou si celle-ci exigeait, pour se consommer, l'action conjointe des deux Institutions. ${ }^{123}$ Or, la rédaction du texte de l'article 290 répond, sur ce point, à un amendement du Groupe du Parti populaire européen, précisément pour permettre à chacune des deux branches du pouvoir législatif de décider de la révocation séparément et d'éviter ainsi des blocages dus à des désaccords entre elles. ${ }^{124}$ Actuellement,

\footnotetext{
${ }^{116}$ Vid. supra, 2.1.1. Cependant, il y a une ambigüité dans l'explication du Præsidium d'après laquelle le texte avait été modifié « pour rendre plus clair que ces conditions sont fixées au cas par cas et ne constituent pas un élément obligatoire », dans CONV 724/03 du 24.5.2003, p. 93.

${ }^{117}$ Cette nouveauté avait déjà été soulignée quant au Traité constitutionnel dans Barón Crespo [7], p. 235. Sur les perspectives de la mise en œuvre par le Parlement européen, cf. Kaeding et Hardacre [28].

${ }^{118}$ Voir l'article 16, paragraphe 3 du Traité sur l'Union européenne et l'article 231 TFUE.

${ }^{119}$ Vid. supra, 2.2.1.

${ }^{120}$ Cette question avait clairement été soulevée et formulée dans un amendement présenté par Dominique de Villepin, en représentation de la France, à la Convention européenne, probablement rejeté en raison du fait qu'il aurait aussi eu pour effet, dans la procédure législative ordinaire, que la révocation n'eut pu être décidée que conjointement par le Parlement européen et par le Conseil.

${ }^{121}$ Voir les articles 8 et 9 du Règlement 973/2010 du Conseil du 25 octobre 2010 portant suspension, à titre temporaire, des droits autonomes du tarif douanier commun à l'importation de certains produits industriels dans les régions autonomes des Açores et de Madère, Journal Officiel L 285 du 30.10.2010, p. 4, p. 5.

${ }^{122}$ Cf. COM (2009) 673 cit., paragraphe 5.1.

${ }^{123}$ Cf. Vírgala Foruria [57], p. 314.

${ }^{124}$ Vid supra, 2.2.2 ; voir aussi amendement à l'article 27 présenté par M. Brok et autres. D'autres amendements ultérieurs en sens contraire ont été présentés, mais ces derniers n'ont pas prospéré.
} 
le Parlement européen, la Commission et la doctrine semblent partager l'approche que « le pouvoir de contrôle peut être exercé par le Parlement Européen et le Conseil chacun de manière autonome $»,{ }^{125}$ confirmée par les clauses type qui visent la révocation dans les actes législatifs. ${ }^{126}$

En ce qui concerne les effets juridiques de la révocation, dans le cas des actes législatifs adoptés suivant la procédure législative ordinaire, elle a comme effet la modification unilatérale de l'acte par le Parlement européen ou par le Conseil. ${ }^{127}$ Mais, si la délégation est révoquée, il conviendra ensuite de rechercher une solution de manière à éviter un vide normatif, soit par des dispositions législatives détaillées, soit par une délégation formulée dans d'autres termes, ce qui ne peut être fait qu'avec la participation des trois Institutions impliquées dans l'activité législative.

Le deuxième mode de contrôle expressément prévu à l'article 290 est le droit d'opposition : l'acte délégué ne peut pas entrer en vigueur «si le Parlement européen ou le Conseil ont exprimé des objections dans le délai fixé par l'acte législatif ». La Commission avait insisté sur le fait que l'opposition «doit être vue comme le mode de contrôle «de droit commun » que le législateur exerce sur l'ensemble des actes délégués », raison pour laquelle elle s'étendait, dans sa Communication, sur les aspects pratiques de ce mode (délais, motifs, effets et même, procédure d'urgence). ${ }^{128}$

Quant à la portée du droit d'opposition, à l'époque du Traité constitutionnel, il était apparu une conception fondée sur le système de délégation en vigueur en Allemagne, qui réduisait ce droit à des « réserves d'information, d'allégation et de consultation », sans possibilité d'entrer dans le fond, sauf pour vérifier que les termes de la délégation avaient été respectés. ${ }^{129}$ Or, cette thèse ne trouve aucun fondement dans la rédaction de l'article 290, qui n'a pas repris les éléments qui limitaient, dans de tels termes, le droit d'opposition du Parlement européen dans la Décision 2006/512 du Conseil. ${ }^{130}$

Quant aux conséquences de l'exercice du droit d'opposition, la Commission a considéré qu'elle pouvait, entre autres options, renoncer à agir, possibilité problématique si l'on tient compte du fait que cela reviendrait à lui reconnaître la liberté d'exercer ou de ne pas exercer les pouvoirs qui lui ont été délégués. En revanche, la Commission ne semble pas revendiquer de droit de retrait des actes délégués avant leur entrée en vigueur lorsqu'il existe une opposition substantielle au sein du Parlement européen ou du Conseil, probablement parce qu'elle considère que les actes

\footnotetext{
${ }^{125}$ Dony [16], p. 198.
}

126 «L'institution qui a entamé une procédure interne afin de décider si elle entend révoquer la délégation de pouvoir s'efforce d'informer l'autre institution et la Commission dans un délai raisonnable avant de prendre une décision finale (...) », p.ex. à l'article 77, paragraphe 2 de la directive 2010/75 relative aux émissions industrielles, Journal Officiel L 334 du 17.12.2010, p. 17 ; p. 48.

${ }^{127}$ Cette conséquence a été relevée, semble-t-il, par le Service juridique du Conseil au cours de la Conférence intergouvernementale préalable au Traité constitutionnel, sans que son intervention n'ait entraîné de modification du texte : cf. Liisberg [32], p. 25.

${ }^{128}$ COM (2009) 673, paragraphes 5.1 et 5.3.

${ }^{129}$ Cf. Rosado [52], p. 369 ; contre Vírgala Foruria [57], p. 315, où il est soutenu que l'interprétation doit se fonder sur le modèle britannique plutôt que sur le modèle allemand.

${ }^{130} \mathrm{La}$ Commission ne partage pas non plus la conception restrictive : cf. COM (2009) 673 cit., paragraphe 5.3.2. 
délégués sont déjà définitifs et non pas de simples propositions ou projets, quand elle les soumet au législateur. ${ }^{131}$

Bien que les modes de contrôle précités permettent l'exercice d'un «contrôle de fer », ${ }^{132}$ il convient de se demander si ce sont les seuls possibles et s'ils peuvent ou non se cumuler.

Certes la Commission n'a indiqué aucun autre mode, ${ }^{133}$ mais, dans l'article 290, ces modes sont précédés d'un «peuvent» dont l'interprétation la plus plausible semble être celle d'une énumération indicative et non pas limitative, d'autant plus que le texte est le fruit d'une modification d'un texte antérieur, suite à la présentation d'amendements tendant justement à éviter cette limitation. ${ }^{134}$ Telle est d'ailleurs la position du Parlement européen, pour qui les mécanismes de contrôles cités à l'article 290, paragraphe 2, «sont purement indicatifs », même s'ils peuvent aussi être considérés comme « les moyens les plus habituels ». ${ }^{135}$

Cela n'implique cependant pas nécessairement la possibilité pour le législateur d'assortir la délégation de clauses d'extinction (sunset clauses), déjà connues dans la législation européenne ${ }^{136}$ et expressément mentionnées dans le premier texte relatif aux actes délégués de la Convention, mais combattues avec succès dans certains amendements $^{137}$ et qui, plus tard, ne seraient pas non plus reprises expressis verbis dans la Décision 2006/512/CE, mais avec certaines acrobaties délicates visant à éviter que celles-ci ne puissent être considérées comme proscrites. ${ }^{138}$ En effet, la validité de telles clauses ne dépend pas seulement de l'interprétation de l'article 290 au vu, notamment des travaux préparatoires, mais aussi d'une appréciation quant à leur conformité, notamment avec le principe de sécurité juridique. Mais les sunset clauses n'épuisent pas les mécanismes de contrôle concevables dans le cadre de l'article $290 ; ;^{139}$ en effet, le Parlement européen a déjà envisagé l'existence d'autres possibilités méritant d'être étudiées, même s'il a aussi reconnu que les mécanismes

${ }^{131}$ Dans la pratique, les actes délégués ne sont publiés au Journal Officiel qu'après l'expiration du délai fixé dans l'acte législatif pour que le Parlement européen ou le Conseil puissent exprimer leurs éventuelles objections.

${ }^{132}$ Andrés Sáenz de Santamaría [1], pp. 205-225 ; p. 223.

${ }^{133} \mathrm{Cf}$. COM (2009) 673 cit., paragraphe 5.1.

${ }^{134}$ Vid. supra, 2.2.2. En revanche, la Convention n'a pas retenu la formule radicale proposée par Mme Paciotti, qui revenait à supprimer toute référence aux mécanismes concrets de contrôle afin de permettre au législateur de les définir librement per casu.

${ }^{135}$ Résolution du 5 mai 2010 cit., paragraphes 2 et 3.

${ }^{136}$ Voir, par exemple, l'article 17 de la Directive 2003/6/CE sur les opérations d'initiés et les manipulations de marché (abus de marché), Journal Officiel L 93 du 12 avril 2003, p. 16.

${ }^{137} \mathrm{La}$ suppression de la référence de ces clauses était fondée sur le fait qu'elles «pourrai[en]t être une source d'incertitude et de problèmes pour la sécurité juridique » : Cf. CONV 724/03 cit. supra, note 112, p. 93, et les amendements à l'article 27 présentés par M. Fayot, M. Fischer, M. Marinho et Mme Van Lanker, ainsi que par M. Santer et d'autres Conventionnels de Luxembourg; dans le sens contraire, renforçant la rédaction, l'amendement de $\mathrm{M}$. Bonde.

${ }^{138}$ Cf. Bradley [9], p. 299.

${ }^{139} \mathrm{C}$ 'est-à-dire, sans préjudice, notamment de la motion de censure prévue dans le Traité et des procédures de contrôle éventuellement prévues dans des procédures législatives spéciales, qui devraient s'appliquer comme lex specialis. 
définis par le législateur doivent respecter certains «principes généraux du droit de l'Union ». ${ }^{140}$

Le respect des principes généraux applicables semble aussi constituer la seule limite juridique à la possibilité de cumuler deux ou plus de deux moyens de contrôle sur un même acte législatif; ainsi, dans la pratique, la révocation se combine avec le droit d'opposition, ${ }^{141}$ suivant les souhaits explicites du Parlement et du Conseil. ${ }^{142}$

En ce qui concerne le rôle des Parlements des États membres, il est clair que les dispositions des Protocoles $n^{0} 1$ et 2 au Traité de Lisbonne s'étendent aux dispositions des actes législatifs qui prévoient une délégation de pouvoir à la Commission, dans les conditions s'avérant applicables auxdits actes. La question est plutôt celle de savoir si les actes délégués, en raison de leurs caractéristiques particulières, doivent ou pas entrer eux aussi dans le champ d'application de ces Protocoles. ${ }^{143}$

La question de la définition de «projet d'acte législatif » à de tels effets a préoccupé la Conférence des organes spécialisés dans les affaires communautaires (COSAC). Dans sa réunion du 4 février 2010, à Madrid, la COSAC a décidé, sur proposition de la délégation britannique, de demander au Conseil et à la Commission si les contrôles prévus dans le Protocole $n^{0} 2$ devaient être appliqués à tous les actes juridiques «dans lesquels participe le Parlement européen » ou aux seuls actes adoptés selon une procédure législative. ${ }^{144}$ Le Conseil comme la Commission avaient répondu dans le sens de la deuxième possibilité, en rappelant l'article 289, paragraphe 3, TFUE, d'après lequel sont législatifs « les actes juridiques adoptés par procédure législative ». ${ }^{145} \mathrm{Or}$, la COSAC a déjà fait part de son «inquiétude » quant à cette thèse, qui à son avis pourrait soustraire du champ d'application du Protocole $\mathrm{n}^{\mathrm{0}} 2$ certains actes « dont le contenu substantiel est législatif ». ${ }^{146}$

Du point de vue strictement juridique, cette inquiétude ne semble pas être fondée, car en l'absence d'une notion particulière d'acte législatif dans les Protocoles 1 et 2, il est clair que les actes que le TFUE considère expressément comme étant des actes non législatifs sont exclus de leur champ d'application. ${ }^{147}$ De plus, il n'est pas clair que cette situation doive changer de iure condendo, compte tenu du rang infra-légal des actes délégués, non expressément reconnu dans le Traité, ${ }^{148}$ mais qui se détache clairement du contexte ${ }^{149}$ et des travaux préparatoires. ${ }^{150}$ Et cela, sans parler des

\footnotetext{
${ }^{140}$ Résolution du 5 mai 2010, cit., paragraphes 2 et 4 .

${ }^{141}$ Voir Règlement UE/438/2010 cit., articles 19 ter et 19 quinquies.

${ }^{142}$ Cf. Résolution du 5 mai 2010 cit., paragraphe 3 ; Doc. 16998/09 du Conseil cit., p. 4.

${ }^{143}$ Cf. Stancanelli [54], pp. 485-528; p. 525.

${ }^{144}$ Lettres de Miguel Arias Cañete aux Présidents du Conseil et de la Commission du 16 février 2010.

${ }^{145}$ Réponse de Maros Sefcovic, Vice-président de la Commission, du 27 avril 2010.

${ }^{146}$ Contribution de la XLIII ${ }^{\mathrm{e}}$ COSAC, Madrid, les 31 mai et $1^{\mathrm{er}}$ juin 2010, paragraphe 9.1., Journal Officiel C 207 du 30.7.2010, p. 1 ; p. 3.

${ }^{147}$ Cf. Pennera [46], pp. 29-48; p. 32 (texte en français); IDEM : «Les Parlements nationaux dans le système de 1'Union européenne »; Rodríguez Iglesias [51], p. 79; pp. 100-101.

${ }^{148}$ Cf. Lenaerts et Desomer [30], pp. 744-765; p. 763 ; Schütze [53], p. 14.

${ }^{149}$ Cf. e.g. Baño Léon [5], p. 203 ; p. 221 ; Andrés Sáenz de Santamariía [2], p. 203 ; p. 221.

${ }^{150} \mathrm{Cf}$. le document Points $\mathrm{n}^{\mathrm{0}} 7$ de la Convention européenne. Certains Conventionnels partisans de donner aux actes délégués un rang de loi avaient présenté des amendements visant à leur donner le nom de «lois
} 
problèmes d'application d'un critère substantiel, entre autres, la question de savoir si l'on devrait distinguer entres les actes délégués qui « complètent » l'acte législatif et ceux qui le «modifient», puisque, en général, une nature matériellement législative (ou quasi-législative) a été vue, surtout quant aux derniers. ${ }^{151}$

\subsubsection{Le contrôle de la Cour de justice et du Tribunal}

Les travaux de la Convention européenne ne laissaient aucun doute sur le fait que les actes délégués seraient soumis au contrôle des juridictions de la Cour de justice de l'Union européenne, ${ }^{152}$ ce qui répondait à un impératif de l'État de droit difficilement contournable au vu tant de la jurisprudence de la Cour de Justice en matière d'actes ultra vires, que des traditions constitutionnelles des États membres en matière de délégation législative. ${ }^{153}$

En ce qui concerne les juridictions compétentes pour connaître des recours, on rappellera, dans les grandes lignes que la Cour de justice est compétente pour connaître des recours en annulation ou en carence formés par des États membres ou par des Institutions de l'Union ou pour statuer, à titre préjudiciel sur des questions soulevées devant des juridictions des États Membres; quant au «Tribunal » (ancien Tribunal de première instance), il est à présent compétent pour connaître de tous les autres recours, sous réserve du pourvoi qui, dans ce cas, peut être formé devant la Cour de justice. $^{154}$

Les recours disponibles ne sont donc pas seulement le recours en annulation prévu à l'article 263 TFUE visé à la Convention européenne dans le contexte des actes délégués, mais aussi, entre autres, le recours en carence visé à l'article 265, qui pourrait avoir un intérêt si la Commission s'abstenait d'exercer les pouvoirs délégués par un acte législatif, contrairement au Traité.

Les actes contre lesquels les recours peuvent être formés sont aussi bien les actes délégués de la Commission que les dispositions portant délégation de l'acte législatif. Par exemple, il est possible d'invoquer une violation de l'article 290 TFUE au motif que, le législateur aurait confié à la Commission la charge de réglementer des «éléments essentiels » de l'acte législatif. D'ailleurs, il faudra voir si, lorsqu'il est allégué que cette limite à la possibilité de déléguer a été violée, la jurisprudence de la Cour de justice circonscrit ou pas son contrôle à l'existence d'une erreur manifeste dans l'appréciation du législateur et, en dans l'affirmative, s'il y aura ou pas des différences dans la méthode employée par la Cour et le Tribunal pour déterminer l'existence d'erreurs «manifestes ».

déléguées » au lieu de «règlements délégués », amendements qui n'avaient pas prospéré : voir l'amendement présenté par M. de Villepin et les amendements suivants de M. Duff et d'autres Conventionnels.

${ }^{151}$ Cf. e.g. Vírgala Foruria [57], p. 304. En revanche, la distinction mentionnée dans le texte ne se trouve pas dans Craig [12], p. 32.

${ }^{152}$ Rapport final du Groupe IX, CONV 424/02 cit. p. 11.

${ }^{153}$ Cf. Diez Picaso [15], pp. 526-539; p. 530.

${ }^{154}$ Art. 256 TFUE en liaison avec l'article 51 du Protocole $\mathrm{n}^{\mathrm{o}} 3$ sur le Statut de la Cour de justice de l'Union européenne. 
En ce qui concerne les recours en annulation formés contre des actes délégués, leur contestation est possible en invoquant l'incompétence de la Commission (actes délégués ultra vires), mais aussi en alléguant l'une des autres causes prévues à l'article 263.

Même si, normalement, il est risqué de vouloir prévoir l'évolution du contentieux des Institutions dans un domaine particulier, l'éventualité de la formation de recours par l'une d'elles dans ce domaine est loin d'être purement hypothétique. Ainsi, dans le cas du Parlement européen, la difficulté que suppose la réunion des voix de la majorité de ses membres pour exercer les droits de révocation et d'opposition ${ }^{155}$ comporte le risque d'un certaine «judiciarisation» des différends avec la Commission. Autre exemple, la position institutionnelle particulière de la Commission pourrait l'amener à saisir la Cour de justice dans le cas où, en conséquence des modifications du texte opérées au cours du processus législatif, celle-ci nourrissait de sérieux doutes sur la conformité au Traité des termes de la délégation adoptés par le législateur.

En ce qui concerne les États membres, on sait qu'ils peuvent être poussés à former des recours en annulation lorsque leurs représentants sont restés en minorité dans le vote du Conseil, soit en raison d'un intérêt particulier dans la matière règlementée, soit en défense de questions de principe. Rien ne nous permet de présumer que cela ne va pas se produire quant aux dispositions portant délégation des actes législatifs.

Quant aux recours formés par les citoyens, il convient de rappeler que le Traité de Lisbonne a considérablement élargi la protection juridictionnelle en déclarant recevables les recours formés à l'encontre d'actes « règlementaires » qui les concernent « directement et qui ne comportent pas de mesures d'exécution ». ${ }^{156}$ D' où l'existence d'un certain débat doctrinal sur l'interprétation de la notion d' "acte règlementaire » contenue dans cet article, qui oppose ceux qui estiment qu'elle comprend toutes sortes d'actes de portée générale et ceux qui considèrent que le recours est limité aux actes de rang infra-légal. ${ }^{157}$

L'intérêt de ce problème quant aux actes délégués réside dans le fait que c'est de lui que dépend la possibilité pour les particuliers de bénéficier de l'ouverture du Traité de Lisbonne, uniquement dans le cadre des recours en annulation contre des actes délégués ou aussi pour contester, par la formation de tels recours, des dispositions portant sur la délégation de l'acte législatif concerné. Or, même en admettant que les termes d' «actes règlementaires » ne sont pas d'une grande clarté dans ce contexte, les travaux préparatoires ne laissent pas l'ombre d'un doute : le qualificatif de «règlementaire » a été introduit délibérément pour circonscrire le changement aux actes juridiques non législatifs de portée générale. ${ }^{158}$

Enfin, en ce qui concerne les recours pour violation du principe de subsidiarité par un acte législatif, formés par les États membres ou par des Parlements ou des chambres parlementaires de ces derniers, ${ }^{159}$ ou encore par le Comité des régions

\footnotetext{
155 vid. supra, 3.2.1.

${ }^{156}$ Article 263, paragraphe 4, TFUE.

${ }^{157}$ Cf. Lenaerts [31], pp. 711-745.

${ }^{158}$ Cf. Di Frederico [14], pp. 13-36; pp. 26-27; Passos [44], pp. 577-580.

${ }^{159}$ Quant au rôle respectif des Gouvernements et des Parlements des Etats membres dans ce contexte cf. Louis [33], p. 429 ; pp. 440-441.
} 
en application de l'article 8 du Protocole $\mathrm{n}^{0} 2$, il semble clair qu'ils permettront de contester les dispositions des actes législatifs qui délèguent à la Commission des pouvoirs normatifs, mais non pas les actes délégués adoptés par la Commission. ${ }^{160}$

\section{Conclusions}

La consécration des actes délégués dans le TFUE ne répond pas à une décision peu réfléchie et n'est pas le reflet d'un défaut de conseil juridique : c'est une solution de synthèse qui combine délibérément des éléments bien connus dans le Droit comparé à d'autres éléments issus de la structure institutionnelle particulière de l'Union ou de la jurisprudence de la Cour de justice, dans l'objet de parvenir, dans chaque cas, à la plus grande légitimité démocratique, compatible avec un exercice efficace du pouvoir normatif. Dans ce sens, elle constitue une concrétisation du principe énoncé à l'article 10, paragraphe 1 , du Traité sur l'Union européenne, d'après lequel «le fonctionnement de l'Union est fondé sur la démocratie représentative ».

Grâce au Traité de Lisbonne, la délégation du pouvoir normatif devient la procédure ordinaire pour l'adoption d'actes non législatifs de portée générale; elle ne doit donc pas être entendue comme l'équivalent de la délégation législative dans les États où l'Exécutif dispose d'un pouvoir réglementaire important. Cependant, la délégation n'occupe pas pour autant tout l'espace que le pouvoir réglementaire prend normalement dans de tels cas; en effet, elle le partage surtout avec des dispositions adoptées par le Conseil en vertu de la réserve de pouvoir normatif dont il continuera à disposer, même si cette réalité reste cachée derrière le fait que, dans de tels cas, le Traité présente les actes du Conseil comme des actes législatifs, puisqu'adoptés par des «procédures législatives spéciales ».

L'autonomie de la Commission dans l'adoption d'actes non législatifs de portée générale ne sera désormais plus gênée par l'intervention de comités composés de représentants des États membres, dont la justification était problématique s'agissant de délégations du législateur dans des domaines relevant, par définition, de la compétence de l'Union, dont l'exercice est en outre soumis au contrôle du respect du principe de subsidiarité. Il serait bon d'éviter que ce progrès soit dévalué dans la pratique en raison des conséquences de la concession de la Commission, de requérir l'assistance de groupes d'experts des États membres, non voulue par la Convention européenne et souhaitée par les Conférences Intergouvernementales, uniquement pour l'exercice du pouvoir délégué dans le domaine particulier des services financiers. Bien qu'un ritorno all'antico semble juridiquement inviable, il conviendra d'évaluer le rôle que ces experts jouent effectivement, d'autant plus que les nostalgiques de la comitologie ne manquent pas. ${ }^{161}$

Grâce à la technique de la délégation de pouvoir du législateur, le Parlement européen acquiert les mêmes pouvoirs que le Conseil pour définir, sur un pied d'égalité

\footnotetext{
${ }^{160}$ Vid. supra, 3.2.1 in fine.

${ }^{161}$ On peut trouver des nostalgiques même dans la doctrine juridique : voir par exemple, pour une position extrêmement circonspecte à l'égard du renforcement du rôle de la Commission dans la délégation de pouvoirs normatifs, Craig [13], pp. 48-64.
} 
dans chaque cas, les limites du pouvoir normatif confié à la Commission, ainsi que pour en superviser l'exercice, non seulement en termes de contrôle de légalité, mais aussi en termes de contrôle d'opportunité. Cette approche conduit à un cadre juridique à la fois cohérent avec le pouvoir de co-législation du Parlement et ayant une vocation apparente de stabilité, puisqu'elle laisse une grande marge pour l'adaptation à l'évolution du contexte général des relations entre le Parlement, le Conseil et la Commission. Il appartient maintenant aux Institutions d'agir dans des conditions d'efficacité ${ }^{162}$ et de transparence, susceptibles d'inspirer la confiance des citoyens.

Enfin, la doctrine juridique pourrait contribuer davantage à une meilleure compréhension des progrès effectués grâce au Traité de Lisbonne si elle parvient à se défaire de certaines inerties. Par exemple, il existe peut-être une raison pour que la classification des actes juridiques fondée sur la catégorie ou le genre d'actes (règlements, directives ou décisions) soit maintenue, dans les présentations des sources de droit, comme la classification principale, au lieu d'être remplacée par la distinction entre actes législatifs et actes non législatifs, désormais lourde de conséquences juridiques et qui est déjà la summa divisio dans la série L du Journal Officiel. Mais elle n'est pas évidente.

\section{Bibliographie}

1. Andrés Sáenz de Santamaría, P.: El sistema institucional en el Tratado de Lisboa : entre la continuidad y el cambio. In: Martín y Pèrez de Nanclares, J. (ed.) El Tratado de Lisboa - La salida de la crisis constitucional. Ed. Iustel, Madrid (2008)

2. Andrés Sáenz de Santamariía, P.: Hacia una Constitución Europea : un balance de los trabajos de la Convención. Rev. Derecho Unión Eur. 6 (2004)

3. Balaguer Callejón, F.: El sistema de fuentes en la Constitución europea. Rev. Derecho Const. Eur. 2 (2004)

4. Balaguer Callejón, F.: La incidencia del Tratado de Lisboa en el sistema de fuentes comunitario y su influencia en los ordenamientos estatales. In: Matia Portilla, F.J. (ed.) Estudios sobre el Tratado de Lisboa. Instituto des Etudios Universitarios/Editorial Comares, Granada (2009)

5. Baño León, J.M.: Las fuentes en el Tratado de la Constitución europea : un análisis prospectivo. Rev. Gen. Derecho Adm. 11 (2006)

6. Baratta, R.: Le "basi giuridiche derivate" nell'ordinamento comunitario. Giustizia Civile (2008)

7. Barón Crespo, E.: Constitucionalización del poder legislativo en la Unión Europea. Civitas, Madrid (2006)

8. Bergström, C.F.: Delegation of Powers in the European Union and the Committee System. Oxford University Press, New York (2005)

9. Bradley, K.St.C.: Delegated Legislation and Parliamentary Supervision in the European Community. In: Epiney, A. et al., Challenging Boundaries : Essays in honour of Roland Bieber, Baden-Baden et St. Gallen (2007)

${ }^{162}$ Lucía Millán a observé qu'à la fin du mois de juillet 2010, aucun acte délégué n'avait encore été adopté, alors que la Commission continuait à adopter des dispositions d'exécution de la législation de l'Union en application des procédures antérieures au Traité de Lisbonne : cf. Milán Moro [37], p. 40, p. 428, note 97. La situation décrite par le Professeur Millán a ensuite évolué (dans ce sens, voir les actes délégués publiés au Journal Officiel L 314 du 30.11.2010), et elle semble d'ailleurs tout à fait normale : s'agissant de la période initiale d'application, il y a eu beaucoup plus de délégations que d'actes délégués; les actes délégués modificatifs se feront attendre et la législation antérieure au Traité de Lisbonne n'a pas encore été adaptée au régime prévu à l'article 290, sauf exception. Les perspectives pour 2011 semblent différentes : voir la Déclaration de la Commission au Journal Officiel L 55 du 28.2.2011, p. 19. 
10. Carrera Hernández, F.J.: Simplificación de los instrumentos jurídicos en el Proyecto de Tratado Constitucional. Rev. Derecho Comunitario Eur. 16 (2007)

11. de Carreras, F.: Por una Constitución Europea. Revista de Estudios Políticos (1995)

12. Craig, P.: The Constitutional Treaty : Legislative and Executive Power in the Emerging Constitutional Order, EUI Working Paper LAW 2004/7, Florence (2004)

13. Craig, P.: The Lisbon Treaty - Law, Politics and Treaty Reform. Oxford (2010)

14. Di Fecerico, G.: The distinction between legislative and non-legislative acts in the Constitutional Treaty and its possible impact on locus standi and non contractual liability. In: Dony, M., Rossi, L.S. (eds.) Démocratie, cohérence et transparence - Vers une constitutionnalisation de l'Union européenne ? Editions de l'Université de Bruxelles, Bruxelles (2008)

15. Diez Picazo, L.M.: Les actes du Gouvernement avec force de loi : une perspective comparée. Annu. Int. Justice Const. 22 (2006)

16. Dony, M.: Droit de l'Union Européenne, 3rd edn. Bruxelles (2010)

17. Duff, A.: The Struggle for Europe's Constitution. Londres, New York (2005)

18. Durand, C.F.: Mieux légiférer : une des priorités centrales de la Commission européenne. ERA Forum $9(2008)$

19. Garzón Clariana, G.: L'inflation normative et le droit communautaire, Journées d'étude à l'occasion du bicentenaire du Code civil, vol. 1. Le rayonnement du droit codifié. Les éditions des Journaux Officiels, Paris (2005)

20. Gil Ibáñez, A.J.: Nueva tipología y jerarquía normativa. Not. Unión Eur. 250 (2005)

21. Guéguen, D.: Comitology-Hijacking European Power? 2nd edn. Bruxelles (2010)

22. Gutiérrez Espada, C., Cervell Hortal, M.J.: La adaptación al Tratado de Lisboa (2007) del sistema institucional decisorio de la Unión, su acción exterior y personalidad jurídica. Grenade (2010)

23. Héritier, A., Moury, C.: Contested delegation : the impact of codecision on Comitology, EUI Working Paper RSCAS 2009/64, Florence (2009)

24. Jacqué, J.P.: Droit institutionnel de l'Union européenne, 6th edn. Paris (2010)

25. Jacqué, J.P.: Le projet de Traité établissant une constitution pour l'Europe - constitutionnalisation ou révision des traités. In: Demaret, P., Govaere, I., Hanf, D. (eds.) 30 ans d'études juridiques européennes au Collège d'Europe - Liber Professorum, Bruxelles (2005)

26. Jiménez Asenjo, R.: El proyecto de "Constitución Europea" y el sistema de fuentes del Derecho. In: Albertí Rovira, E. (ed.) El proyecto de nueva Constitución Europea, Valencia (2004)

27. Joerges, C., Vos, E. (eds.): EU Committees : Social Regulation, Law and Politics. Oxford (1999)

28. Kaeding, M., Hardacre, A.: The execution of delegated powers after Lisbon. A timely analysis of the regulatory procedure with scrutiny and its lessons for delegated acts, EUI Working Papers, RSCAS 2010/85, Florence (2010)

29. Kellermann, A.E., et al.: directeurs : Improving the Quality of Legislation in Europe. La Haye (1998)

30. Lenaerts, K., Desomer, M.: Towards a hierarchy of legal acts in the European Union ? Simplification of legal instruments and procedures. Eur. Law J. 11 (2005)

31. Lenaerts, K.: Le Traité de Lisbonne et la protection juridictionnelle des particuliers en droit de l'Union, Cahiers de droit européen XLV (2009)

32. Liisberg, J.G.: The EU Constitutional Treaty and its distinction between legislative and non-legislative acts - oranges into apples ? Jean Monnet Working Paper 01/06, New York (2006)

33. Louis, J.-V.: The Lisbon Treaty : The Irish 'No'. National parliaments and the principle of subsidiarity - legal options and practical limits. Eur. Const. Law Rev. 4 (2008)

34. Mangas Martín, A.: Algunos aspectos del Derecho derivado en el Tratado de Lisboa : categorización de los actos, indeterminación de los tipos de actos, bases jurídicas y jerarquía. Rev. Gen. Derecho Eur. (2009)

35. Masson, A.: Les conditions de validité des bases juridiques dérivées à la suite de l'arrêt du 6 mai 2006. Cah. Droit Eur. 44 (2008)

36. Méndez de Vigo, I.: El rompecabezas - Así redactamos la Constitución Europea. Madrid (2005)

37. Milán Moro, L.: 1 ordenamiento comunitario, del Tratado Constitucional al Tratado de Lisboa. Rev. Derecho Comunitario Eur. 36 (2010)

38. Moreiro González, C.J.: El ocaso de la "comitología" ? Revista de Derecho Comunitario Europeo (13) (2002)

39. Navarro Batista, N.: ases jurídicas derivadas, delegación legislativa y poder de ejecución : el equilibrio institucional en la sentencia del TJUE de 6 de mayo de 2008, Parlamento Europeo c. Consejo. Rev. Gen. Derecho Eur. 18 (2009)

40. Neergard, A.: La comitologie et la légitimité démocratique de la Communauté européenne. Schoo, J., Pennera, C. (eds.) Liber amicorum en l'honneur de Gregorio Garzón Clariana, inédit (2006) 
41. Neuhold, C.: Taming the "Trojan Horse" of Comitology? Accountability issues of Comitology and the Role of the European Parliament. Eur. Integr. Online Papers 12 (2008)

42. Norman, P.: The Accidental Constitution - The Story of the European Convention. Bruxelles (2003)

43. Otto, I.: Derecho constitucional-Sistema de fuentes, 2nd edn. 7ème réimpression. Barcelone (1999)

44. Passos, R.: Le système juridictionnel de l'Union; Amato, G., Bribosia, H., de Witte, B. : Genèse et destinée de la Constitution européenne - Genesis and Destiny of the European Constitution. Bruxelles (2007)

45. Pennera, C.: Les enjeux du Traité modificatif. ERA Forum 9 (2008)

46. Pennera, C.: Algunes reflexions sobre el principi de subsidiarietat al Tractat de Lisboa, PARLEMENT DE LA CATALOGNE : El control del principi de subsidiarietat. Barcelone (2010) (in French)

47. Pérez Royo, J.: Las fuentes del Derecho, 5th edn., réimpression. Madrid (2008)

48. Piris, J.C.: The Constitution for Europe-A Legal Analysis. Cambridge (2006)

49. Piris, J.-C.: The Lisbon Treaty - A Legal and Political Analysis. Cambridge (2010)

50. Ponzano, P.: "Executive" and "delegated" acts : the situation after the Lisbon Treaty. In: Griller, S., Ziller, J. (eds.) The Lisbon Treaty - EU Constitutionalism Without a Constitutional Treaty ?, Vienne (2008)

51. Rodríguez Iglesias, G.C., Ortiz Blanco, L.: The Role of National Parliaments in the European Union. Madrid (2010)

52. Rosado Pacheco, S.: Los Reglamentos delegados en el Tratado por el que se establece una Constitución para Europa. In: ÁlvarezConde, E., Garrido Mayol, V. (eds.) Comentarios a la Constitución Europea, Valencia (2004)

53. Schütze, R.: Sharpening the Separation of Powers through a Hierarchy of Norms ? Reflections on the Draft Constitutional Treaty's regime for legislative and executive law-making, EIPA Working Paper W/01 (2005)

54. Stancanelli, P.: Le système décisionnel de l'Union. In: Amato, G., Bribosia, H., De Witte, B. (eds.) Genèse et destinée de la Constitution européenne, Bruxelles (2007)

55. Szapiro, M.: Comitologie : rétrospective et prospective après la réforme de 2006. Revue du Droit de l'Union européenne (3) (2006)

56. Vedaschi, A.: Esistono atti comunitari di "terzo tipo"? Diritto pubblico comparato ed europeo (2002)

57. Vírgala Foruria, E.: Los actos no legislativos en la Constitución Europea. Rev. Derecho Const. Eur. 6 (2006) 